\title{
ON THE CR POINCARÉ-LELONG EQUATION, YAMABE STEADY SOLITONS AND STRUCTURES OF COMPLETE NONCOMPACT SASAKIAN MANIFOLDS
}

\author{
*DER-CHEN CHANG, ${ }^{* *}$ SHU-CHENG CHANG, ${ }^{\dagger}$ YINGBO HAN, AND CHIEN LIN
}

\begin{abstract}
In this paper, we solve the so-called CR Poincaré-Lelong equation by solving the CR Poisson equation on a complete noncompact $\mathrm{CR}(2 n+1)$-manifold with nonegative pseudohermitian bisectional curvature tensors and vanishing torsion which is an odd dimensional counterpart of Kähler geometry. With applications of this solution plus the CR Liouvelle property, we study the structures of complete noncompact Sasakian manifolds and CR Yamabe steady solitons.
\end{abstract}

\section{INTRODUCTION}

It is conjectured ( $[\mathrm{GW1}, \underline{\underline{\mathrm{S}}}]$ and $[\mathrm{Y}]$ ) that a complete noncompact Kahler manifold of complex dimension $m$ with positive holomorphic bisectional curvature is biholomorphic to $\mathbf{C}^{m}$. It is the very first result concerning such a conjecture was obtained by Mok-Siu-Yau ([MSY]) and Mok ([Mok2]). More precisely, they proved that a complete noncompact Kähler manifold of nonnegative holomorphic bisectional curvature $M$ is isometrically biholomorphic to $\mathbf{C}^{m}, m \geq 2$ under the assumptions of the maximum volume growth condition

$$
V_{o}(r) \geq \delta r^{2 m}
$$

for some point $o \in M, \delta>0, r(x)=d(o, x)$ and the scalar curvature $R$ decays as

$$
R(x) \leq \frac{C}{1+r^{2+\varepsilon}}, \quad x \in M
$$

for $C>0$ and any arbitrarily small positive constant $\varepsilon$. A Riemannian version of ([MSY]) was proved in GW2 shortly afterwards. Since then there are several further works aiming to prove the optimal result and

1991 Mathematics Subject Classification. Primary 32V05, 32V20; Secondary 53C56.

Key words and phrases. CR Poisson equation, CR Poincaré-Lelong equation, CR Yamabe solitons.

*Der-Chen Chang is partially supported by an NSF grant DMS-1408839 and a McDevitt Endowment Fund at Georgetown University.

** Shu-Cheng Chang is partially supported in part by the MOST of Taiwan.

†Yingbo Han is partially supported by an NSFC grant No. 11201400, Nanhu Scholars Program for Young Scholars of Xinyang Normal University and the Universities Young Teachers Program of Henan Province (2016GGJS-096), China Scholarship Council(201508410400). 
reader is referred to [Mok1, [CTZ, [CZ], [N2], [NT1, [NT2] and [NST]. A key common ingredient used in the previous works such as $\mathrm{MSY}$, [N2] and [NT2] is to solve the so-called Poincaré-Lelong equation

$$
i \partial \bar{\partial} u=\rho,
$$

by first solving the Poisson equation

$$
\Delta u=\operatorname{trace}(\rho)
$$

for a given Ricci form $\rho$ of $M$. They then applied the results to study the analytic and geometric properties of $M$. Note that we refer to [N1] and [CF] for adapting a different method which has also succeeded in the recent resolution of the fundamental gap conjecture.

A CR $(2 n+1)$-manifold of vanishing pseudohermitian torsion (i. e. Sasakian manifold) is an odd dimensional counterpart of Kähler geometry, then it is very natural to concerned with an CR analogue of Mok-Siu-Yau type theorem of Poincare-Lelong equation in a complete noncompact strictly pseudoconvex CR $(2 n+1)$-manifold of vanishing pseudohermitian torsion with nonnegative pseudohermitian bisectional curvature.

Let $(M, J, \theta)$ be a complete noncompact strictly pseudoconvex CR $(2 n+1)$-manifold (see in the appendix for basic notions). A piecewise smooth curve $\gamma:[0,1] \rightarrow M$ is said to be horizontal if $\dot{\gamma}(t) \in \mathcal{D}$ whenever $\dot{\gamma}(t)$ exists. Here $\mathcal{D}$ is the linear span of the tangential vector fields in $\left\{Z_{\alpha}, Z_{\bar{\alpha}}\right\}_{\alpha=1}^{n}$ which is a $2 n$-dimensional vector space. We denote $C_{p, q}$ to be the set of all horizontal curves joining $p, q$. Since $M$ is strictly pseudoconvex, the Levi form is positive definite. This implies that $\mathcal{D} \cup[\mathcal{D}, \mathcal{D}]=T M$, the tangent bundle of $M$. Therefore, $C_{p, q} \neq \emptyset$ by Chow's theorem (see e.g., $[\mathrm{CH}]$ and $[\mathrm{CaC}$ ). The Carnot-Carathéodory distance between two points $p, q \in M$ is defined by

$$
d_{c c}(p, q)=\inf \left\{l(\gamma): \gamma \in C_{p, q}\right\}
$$

where the length of $\gamma$ is

$$
l(\gamma)=\int_{0}^{1}\langle\dot{\gamma}(t), \dot{\gamma}(t)\rangle_{L_{\theta}}^{\frac{1}{2}} d t .
$$

Now given any nonnegative function $f$ on $M$, we define

$$
k_{f}(x, t)=\frac{1}{V_{x}(t)} \int_{B_{x}(t)} f d \mu .
$$

Here $V_{x}(r)$ is the volume of the geodesic ball $B_{x}(r)$ with respect to the Carnot-Carathéodory distance $r(x, y)$ between $x$ and $y$, and $r(x)=r(x, o)$ where $o \in M$ is a fixed point. Next we give the definition of the 
sublaplacian $\triangle_{b}$ (see the formula (A.1)).

$$
\Delta_{b} u=\operatorname{Tr}\left(\left(\nabla^{H}\right)^{2} u\right)=\sum_{\alpha}\left(u_{\alpha \bar{\alpha}}+u_{\bar{\alpha} \alpha}\right) .
$$

We will follows the method of solving Poisson equation as in [NST].

Theorem 1.1. Let $(M, J, \theta)$ be a complete noncompact strictly pseudoconvex $C R(2 n+1)$-manifold with nonnegative pseudohermitian Ricci curvature tensors and vanishing torsion. Let $f$ be a nonnegative function and $k(t)=k_{f}(o, t)$, where $o \in M$ is a fixed point. Suppose that

$$
\int_{0}^{\infty} k(t) d t<\infty
$$

and suppose that there exist $1>\delta>0, h(t) \geq 0,0 \leq t \leq \infty$ with $h(t)=o(t)$ as $t \rightarrow \infty$ such that

$$
\int_{0}^{t} s k(x, t) d s \leq h(t)
$$

for all $x$ and for all $t \geq \delta r(x)$. Then the CR Poisson equation

$$
\triangle_{b} u=f
$$

has a solution such that for all $1>\varepsilon>0$

$$
\begin{aligned}
\alpha_{1} r \int_{2 r}^{\infty} k(t) d t+\beta_{1} \int_{0}^{2 r} t k(t) d t & \geq u(x) \\
& \geq-\alpha_{2} r \int_{2 r}^{\infty} k(t) d t-\beta_{2} \int_{0}^{\varepsilon r} t k(x, t) d t+\beta_{3} \int_{0}^{2 r} t k(t) d t
\end{aligned}
$$

for some positive constants $\alpha_{1}, \beta_{i}, 1 \leq i \leq 3$. which depend on $n$ and $\alpha_{2}$ which depends on $n, \varepsilon$.

The main consequence is that if $f$ decays faster than $r^{-1}$ in the following sense, then (1.3) has a solution.

Corollary 1.1. Let $(M, J, \theta)$ be a complete noncompact strictly pseudoconvex $C R(2 n+1)$-manifold with nonnegative pseudohermitian Ricci curvature tensors and vanishing torsion. Let $f$ be a nonnegative function and $k(t)=k_{f}(o, t)$, where $o \in M$ is a fixed point. Suppose that

$$
\int_{0}^{\infty} k(t) d t<\infty
$$

and

$$
\sup _{\partial B_{o}(r)} f=o\left(r^{-1}\right)
$$

as $r \rightarrow \infty$. Then the CR Poisson equation

$$
\triangle_{b} u=f
$$

has a solution. 
By deriving some basic results for solutions to the CR heat equation on complete noncompact pseudohermitian $(2 n+1)$-manifolds, we are able to solve the CR Poincaré-Lelong equation with the help of Theorem 1.1

The key is, following from Corollary 2.1 with $f=\operatorname{trace}(\rho)$ and $f_{0}(x)=0$, that $u_{0}(x)=0$ and then (4.3) ( in section 4) reduces to the CR Poisson equation (1.3).

Theorem 1.2. Let $(M, J, \theta)$ be a complete $C R(2 n+1)$-manifold with nonnegative pseudohermitian bisectional curvature and vanishing torsion. Let $\rho$ be a real closed $(1,1)$ form with the nonnegative trace $f$ and $\rho_{\alpha \bar{\beta}, 0}=0$, for $\alpha, \beta=1, \cdots, n$. Assume that

$$
\int_{0}^{\infty} \frac{1}{V\left(B_{0}(t)\right)} \int_{B_{0}(t)}\|\rho\| d \mu d t<\infty
$$

and

$$
\lim \inf _{r \rightarrow \infty}\left[\exp \left(-a r^{2}\right) \int_{B_{0}(r)}\|\rho\|^{2} d \mu\right]<\infty, \text { for some } a>0 .
$$

Then there is a solution of the CR Poincaré- Lelong equation

$$
i \partial_{b} \bar{\partial}_{b} u=\rho .
$$

Moreover for any $0<\varepsilon<1$, u satisfies

$$
\begin{aligned}
\alpha_{1} r \int_{2 r}^{\infty} k(t) d t+\beta_{1} \int_{0}^{2 r} t k(t) d t & \geq u(x) \\
& \geq-\alpha_{2} r \int_{2 r}^{\infty} k(t) d t-\beta_{2} \int_{0}^{\varepsilon r} t k(x, t) d t+\beta_{3} \int_{0}^{2 r} t k(t) d t
\end{aligned}
$$

for some positive constants $\alpha_{1}, \beta_{i}, 1 \leq i \leq 3$. which depend on $n$ and $\alpha_{2}$ which depends on $n, \varepsilon$.

With its applications plus the CR Liouvelle theorem, we have

Corollary 1.2. Let $(M, J, \theta)$ be a complete $C R(2 n+1)$-manifold with nonnegative pseudohermitian bisectional curvature and vanishing torsion. Let $\rho$ be a nonnegative real closed $(1,1)$ form and $\rho_{\alpha \bar{\beta}, 0}=0$. Then $\rho \equiv 0$ if the $\rho$ satisfies

$$
\int_{0}^{r} s\left[\frac{1}{V\left(B_{0}(t)\right)} \int_{B_{0}(t)}\|\rho\| d \mu\right] d s=o(\log r)
$$

$$
\lim \inf _{r \rightarrow \infty}\left[\exp \left(-a r^{2}\right) \int_{B_{0}(r)}\|\rho\|^{2} d \mu\right]<\infty
$$


Finally, we are able to investigate the geometry and classification of $(2 n+1)$-dimensional CR Yamabe solitons $\left(M^{2 n+1}, \theta, f, \mu\right)$ satisfying the soliton equation which is a special class of solutions to the CR Yamabe flow

$$
\left\{\begin{array}{l}
\frac{\partial}{\partial t} \theta(t)=-2 W(t) \theta(t), \\
\theta(0)=\grave{\theta}
\end{array}\right.
$$

on a pseudohermitian $(2 n+1)$-manifold $\left(M^{2 n+1}, \stackrel{\circ}{\theta}\right)$ given by $(\operatorname{cf}[\mathrm{CCC}])$, where $W(t)$ is the Tanaka-Webster scalar curvature with respect to the evolving contact form $\theta(t)$. Moreover, this special class of solutions to the CR Yamabe flow (1.10) is given by self-similar solutions, whose contact forms $\theta_{t}$ deform under the CR Yamabe flow only by a scaling function depending on $t$ and reparametrizations by a 1-parameter family of contact diffeomorphisms; meanwhile, the CR structure $J$ shall be invariant under these diffeomorphisms.

Definition 1.1. ([CCC] $)(i)$ We call $\left(M^{2 n+1}, \theta, f, \mu\right)$ a complete $(2 n+1)$-dimensional CR Yamabe soliton if for a family $\theta_{(t)}$ of contact forms on $(M, \stackrel{\theta}{)}$ evolving by the $C R$ Yamabe flow (1.10) on $M \times[0, T)$ with the maximal time $T$ and for any pairs $t_{1}<t_{2}$, the contact forms $\theta_{\left(t_{1}\right)}$ and $\theta_{\left(t_{2}\right)}$ differ only by a contact diffeomorphism $\Phi$ with $\theta_{\left(t_{2}\right)}=\rho(t) \Phi^{*} \theta_{\left(t_{1}\right)}$ for some smooth function $\rho(t)$ with $\rho(t)>0$ and $\rho(0)=1$. Furthermore, one can have a smoth function $f$ and a constant $\mu=-\frac{1}{2} \rho^{\prime}(0)$ such that

$$
\left\{\begin{aligned}
W \theta+\frac{1}{2} \mathrm{~L}_{X_{f}} \theta & =\mu \theta, \\
f_{\alpha \alpha}+i A_{\alpha \alpha} f & =0,
\end{aligned}\right.
$$

Here $\mathrm{L}_{X_{f}}$ denotes Lie derivative by $X_{f}$ with $L_{X_{f}} J \equiv 2\left(f_{\alpha \alpha}+i A_{\alpha \alpha} f\right) \theta^{\alpha} \otimes Z_{\bar{\alpha}}+2\left(f_{\bar{\alpha} \bar{\alpha}}-i A_{\bar{\alpha} \bar{\alpha}} f\right) \theta^{\bar{\alpha}} \otimes Z_{\alpha} \bmod \theta$ and $X_{f}=i f_{\alpha} Z_{\bar{\alpha}}-i f_{\bar{\alpha}} Z_{\alpha}-f \mathbf{T}$. That is

$$
f_{0}+2 W=2 \mu
$$

with $f_{\alpha \alpha}+i A_{\alpha \alpha} f=0$. It is called shrinking if $\mu>0$, steady if $\mu=0$, and expanding if $\mu<0$.

(ii) A complete $(2 n+1)$-dimensional CR Yamabe soliton is called a complete gradient CR Yamabe soliton if there exists a smooth function $u$ on $M$ such that

$$
\Delta_{b} u=2(W-\mu)
$$

which is the same as

$$
\Delta_{b} u=-f_{0}
$$

Remark 1.1. Let $\left(\mathbf{H}^{n}, J, \theta, u, \mu\right)$ be a Heisenberg $(2 n+1)$-manifold with $W=0$ and $A_{\alpha \beta}=0$. It is a Gaussian-type gradient CR Yamabe soliton for $u=\mu|z|^{2}$ with $\mu=-1,0,1$. 
In particular, every closed CR Yamabe soliton is a gradient CR Yamabe soliton (1.11). In general, it follows from Corollary 1.1 that

Corollary 1.3. Any complete noncompact steady CR Yamabe soliton with nonnegative pseudohermitian Ricci curvature tensors and vanishing torsion is a complete steady gradient CR Yamabe soliton if

$$
\int_{0}^{\infty} k(t) d t<\infty
$$

and

$$
\sup _{\partial B_{o}(r)} W=o\left(r^{-1}\right)
$$

as $r \rightarrow \infty$. Here $W(x)$ is the nonnegative Tanaka-Webster scalar curvature and

$$
k(x, t)=\frac{1}{V_{x}(t)} \int_{B_{x}(t)} W d \mu
$$

As a consequence of Corollary 1.2 with $\rho_{\alpha \bar{\beta}}=R_{\alpha \bar{\beta}}$, together with the Liouvelle property Lemma 5.1 we are able to investigate the structure of complete noncompact CR $(2 n+1)$-manifolds of nonnegative pseudohermitian bisectional curvature and vanishing torsion (i.e. Sasakian manifolds) and complete steady CR Yamabe solitons as well. Note that by CR Bianchi identity ([L1]), we have $R_{\alpha \bar{\beta}, 0}=0$ in a CR manifold with vanishing torsion.

Corollary 1.4. Let $\left(M^{2 n+1}, J, \theta, \varphi, \mu\right)$ be a complete noncompact $C R(2 n+1)$-manifold of nonnegative pseudohermitian bisectional curvature and vanishing torsion. Assume that

$$
\int_{0}^{r} s\left(\frac{1}{V\left(B_{0}(t)\right)} \int_{B_{0}(t)}\|R i c\| d \mu\right) d s=o(\log r)
$$

$$
\lim \inf _{r \rightarrow \infty}\left[\exp \left(-a r^{2}\right) \int_{B_{0}(r)}\|R i c\|^{2} d \mu\right]<\infty
$$

Then $M$ must be the CR flat space form. In particular any complete steady CR Yamabe soliton of nonnegative pseudohermitian bisectional curvature and vanishing torsion with (1.13) and 1.14) must be the CR flat space form.

Remark 1.2. In the paper of $[\mathrm{CCC}]$, the second named author obtained the structure of complete 3dimensional pseudo-gradient CR Yamabe solitons (shrinking, or steady, or expanding) of vanishing torsion. 
The rest of the paper is organized as follows. In section 2 , we will discuss the condition on $f$ so that $\triangle_{b} u=f$ has a solution $u$ and we also discuss the properties of $u$. In section 3 , we derive some basic results for solutions to the $\mathrm{CR}$ heat equation on complete noncompact pseudohermitian $(2 n+1)$-manifolds. In section 4, we solve the CR Poincaré-Lelong equation by first solving the CR Poisson equation as in section 2. In section 5 , we are able to investigate the structure of complete noncompact CR $(2 n+1)$-manifolds of nonnegative pseudohermitian bisectional curvature and vanishing torsion (Sasakian) and complete steady CR Yamabe solitons as well.

Remark 1.3. In this paper, the CR heat equation is for the sublaplacian

$$
\triangle_{b}=\sum_{\alpha=1}^{n}\left(Z_{\alpha} Z_{\bar{\alpha}}+Z_{\bar{\alpha}} Z_{\alpha}\right)
$$

not for the Kohn Laplacian $\square_{b}$. However, the result for $\triangle_{b}$ can be extended easily to the the Kohn Laplacian (at least on $\left(0,1\right.$-forms) $\square_{b}=\triangle_{b}+i \beta T$ with $|\beta|<1$. Then we may use meromorphic extension to deal with other $\beta \in \mathbf{C} \backslash \mathcal{E}$ where $\mathcal{E}$ is a discrete exceptional set. See e.g., [CCFI].

\section{The CR Poisson Equation}

Let $(M, J, \theta)$ be a complete noncompact strictly pseudoconvex CR $(2 n+1)$-manifold with nonnegative pseudohermitian Ricci curvature tensors and vanishing torsion. Let $f$ be a nonnegative function on $M$. In this section, we will discuss the condition on $f$ so that $\triangle_{b} u=f$ has a solution $u$ and we also discuss the properties of $u$.

Let $H(x, y, t)$ be the heat kernel of the CR heat equation on $M$, we define the Green function

$$
G(x, y)=\int_{0}^{\infty} H(x, y, t) d t
$$

if the integral on the right side converges. We can checks that $G$ is positive and $\triangle_{b} G(x, y)=-\delta_{x}(y)$. It follows from the properties of heat kernel and Volume double property ([CCHT] and $[\mathrm{BBG}]$ ) that we obtain the following result :

Theorem 2.1. Let $(M, J, \theta)$ be a complete noncompact strictly pseudoconvex $C R(2 n+1)$-manifold with nonnegative pseudohermitian Ricci curvature tensors and vanishing torsion. Then we have

$$
C_{1} \int_{r^{2}(x, y)}^{\infty} V_{x}^{-1}(\sqrt{t}) d t \leq G(x, y) \leq C_{2} \int_{r^{2}(x, y)}^{\infty} V_{x}^{-1}(\sqrt{t}) d t
$$

where $C_{1}, C_{2}$ are two positive constants which depend $n$. 
Proof. It follows from the definition of the Green function $G(x, y)$, we have

$$
G(x, y)=\int_{0}^{\infty} H(x, y, t) d t=\int_{0}^{r^{2}} H(x, y, t) d t+\int_{r^{2}}^{\infty} H(x, y, t) d t
$$

a) If $t \geq r^{2}(x, y)$, it follows from [CCHT, Proposition 3.1.] that

$$
H(x, y, t) \leq C_{3} V^{-\frac{1}{2}}\left(B_{x}(\sqrt{t})\right) V^{-\frac{1}{2}}\left(B_{y}(\sqrt{t})\right) \exp \left(-C_{4} \frac{r^{2}(x, y)}{t}\right)
$$

where $C_{3}, C_{4}$ are positive constants. Moreover, from the CR volume doubling property ([CCHT, Corollary 1.1.]),

$$
V\left(B_{x}(\sqrt{t})\right) \leq V\left(B_{y}(\sqrt{t}+r)\right) \leq V\left(B_{y}(2 \sqrt{t})\right) \leq C_{5} 2^{2 C_{6}} V\left(B_{y}(\sqrt{t})\right)
$$

where $C_{5}$ is a positive constant which depends only on $n$. Thus

$$
V^{-\frac{1}{2}}\left(B_{y}(\sqrt{t})\right) \leq C_{5}^{\frac{1}{2}} 2^{C_{6}} V^{-\frac{1}{2}}\left(B_{x}(\sqrt{t})\right)
$$

It follows from (2.2) and (2.3) that

$$
H(x, y, t) \leq C_{7} V^{-1}\left(B_{x}(\sqrt{t})\right)
$$

and then

$$
G(x, y) \leq \int_{0}^{r^{2}} H(x, y, t) d t+C_{7} \int_{r^{2}}^{\infty} V^{-1}\left(B_{x}(\sqrt{t})\right) d t .
$$

b) If $t \leq r^{2}(x, y)$, again from [CCHT, Proposition 3.1.], we have

$$
V\left(B_{x}(\sqrt{t})\right) \leq V\left(B_{y}(r+\sqrt{t})\right) \leq C_{5}\left(1+\frac{r}{\sqrt{t}}\right)^{2 C_{6}} V\left(B_{y}(\sqrt{t})\right)
$$

that is,

$$
V^{-\frac{1}{2}}\left(B_{y}(\sqrt{t})\right) \leq C_{5}^{\frac{1}{2}}\left(1+\frac{r}{\sqrt{t}}\right)^{C_{6}} V^{-\frac{1}{2}}\left(B_{x}(\sqrt{t})\right) .
$$

Thus

$$
H(x, y, t) \leq C_{3} C^{\frac{1}{2}}\left(1+\frac{r}{\sqrt{t}}\right)^{C_{6}} V^{-1}\left(B_{x}(\sqrt{t})\right) \exp \left(-C_{4} \frac{r^{2}}{t}\right) .
$$

Since $\left(1+\frac{r}{\sqrt{t}}\right) \leq C_{8} \exp \left(\frac{C_{4}}{2} \frac{r^{2}}{t}\right)$, where $C_{8}$ is a positive constant depending $C_{6}$, we have

$$
H(x, y, t) \leq C_{9} V^{-1}\left(B_{x}(\sqrt{t})\right) \exp \left(-\frac{C_{4}}{2} \frac{r^{2}}{t}\right),
$$

where $C_{9}$ is a positive constant which depends only on $n$. Letting $s=\frac{r^{4}}{t}$ with $r^{2} \leq s$, we have

$$
\begin{aligned}
\int_{0}^{r^{2}} H(x, y, t) d t & \leq C_{9} \int_{0}^{r^{2}} V^{-1}\left(B_{x}(\sqrt{t})\right) \exp \left(-\frac{C_{4}}{2} \frac{r^{2}}{t}\right) d t \\
& =C_{9} \int_{r^{2}}^{\infty} V^{-1}\left(B_{x}\left(\frac{r^{2}}{\sqrt{s}}\right)\right) \exp \left(-\frac{C_{4}}{2} \frac{s}{r^{2}}\right) \frac{r^{4}}{s^{2}} d s
\end{aligned}
$$


Again from [CCHT, Proposition 3.1.], we have

$$
V\left(B_{x}(\sqrt{s})\right)=V\left(B_{x}\left(\frac{s}{r^{2}} \frac{r^{2}}{\sqrt{s}}\right)\right) \leq C_{5}\left(\frac{s}{r^{2}}\right)^{2 C_{6}} V\left(B_{x}\left(\frac{r^{2}}{\sqrt{s}}\right)\right),
$$

that is,

$$
V^{-1}\left(B_{x}\left(\frac{r^{2}}{\sqrt{s}}\right)\right)=V\left(B_{x}\left(\frac{s}{r^{2}} \frac{r^{2}}{\sqrt{s}}\right)\right) \leq C_{5}\left(\frac{s}{r^{2}}\right)^{2 C_{6}} V^{-1}\left(B_{x}(\sqrt{s})\right) .
$$

From (2.8) and (2.9), we have

$$
\int_{0}^{r^{2}} H(x, y, t) d t \leq C_{9} C_{5} \int_{r^{2}}^{\infty} V^{-1}\left(B_{x}(\sqrt{s})\right)\left(\frac{r^{2}}{s}\right)^{2-2 C_{6}} \exp \left(-\frac{C_{4}}{2} \frac{s}{r^{2}}\right) d s .
$$

However, the function $x^{2 C_{6}-2} \exp \left(-\frac{C_{4}}{2} x\right)$ is bound from above. So we have

$$
\int_{0}^{r^{2}} H(x, y, t) d t \leq C_{10} \int_{r^{2}}^{\infty} V^{-1}\left(B_{x}(\sqrt{t})\right) d t
$$

where $C_{10}$ is a positive constant which depends only on $n$. Finally it follow from (2.5) and (2.11) that

$$
G(x, y) \leq C_{2} \int_{r^{2}}^{\infty} V^{-1}\left(B_{x}(\sqrt{t})\right) d t
$$

where $C_{2}$ is a positive constant which depends only on $n$. By using the low bound of heat kernel, volume doubling property as in $[\mathrm{CCHT}]$ and $[\mathrm{BBG}$, we can also prove

$$
G(x, y) \geq C_{1} \int_{r^{2}}^{\infty} V^{-1}\left(B_{x}(\sqrt{t})\right) d t
$$

where $C_{1}$ is a positive constant which depends only on $n$.

Theorem 2.2. Let $(M, J, \theta)$ be a complete noncompact strictly pseudoconvex $C R(2 n+1)$-manifold with nonnegative pseudohermitian Ricci curvature tensors and vanishing torsion. Assume $(M, J, \theta)$ is nonparabolic and there is a constant $\sigma>0$ such that the minimal Green's function $G(x, y)$ satisfies

$$
\sigma^{-1} \frac{r^{2}(x, y)}{V\left(B_{x}(r(x, y))\right)} \leq G(x, y) \leq \sigma \frac{r^{2}(x, y)}{V\left(B_{x}(r(x, y))\right)}
$$

for all $x \neq y$ in $M$. Let $f$ be a nonnegative function and let $k(x, t)=k_{f}(x, t)$ and $k(t)=k(o, t)$, where $o \in M$ is a fixed point. Suppose that

$$
\int_{0}^{\infty} k(t) d t<\infty
$$

Then the CR Poisson equation

$$
\triangle_{b} u=f
$$


has a solution $u$ such that for all $1>\varepsilon>0$,

$$
\begin{aligned}
\alpha_{1} \int_{2 r}^{\infty} k(t) d t+\beta_{1} \int_{0}^{2 r} k(t) d t & \geq u(x) \\
& \geq-\alpha_{2} r \int_{2 r}^{\infty} k(t) d t-\beta_{2} \int_{0}^{\varepsilon r} t k(x, t) d t+\beta_{3} \int_{0}^{2 r} t k(t) d t
\end{aligned}
$$

for some positive constants $\alpha_{1}, \alpha_{2}, \beta_{i}, 1 \leq i \leq 3$.

Proof. It follows from (2.1) and (2.12) that

$$
C^{-1} \frac{r^{2}(x, y)}{V\left(B_{x}(r(x, y))\right)} \leq \int_{r}^{\infty} \frac{t}{V\left(B_{x}(t)\right)} d t \leq C \frac{r^{2}(x, y)}{V\left(B_{x}(r(x, y))\right)}
$$

where $C$ is positive constant which depends on $n$ and $\sigma$. For all $R>0$, let $G_{R}$ be the positive Green's function on $B_{o}(R)$ with zero boundary value and denote

$$
u_{R}(x)=\int_{B_{o}(R)}\left(G_{R}(o, y)-G_{R}(x, y)\right) f(y) d y
$$

Then

$$
\triangle_{b} u=f
$$

in $B_{o}(R)$ and $u_{R}(o)=0$. For any $x$ with $r(x)=r$ with $R \gg r$, then

$$
u_{R}(x)=I+I I
$$

with

$$
I=\int_{B_{o}(R) \backslash B_{o}(2 r)}\left(G_{R}(o, y)-G_{R}(x, y)\right) f(y) d y
$$

and

$$
I I=\int_{B_{o}(2 r)}\left(G_{R}(o, y)-G_{R}(x, y)\right) f(y) d y .
$$

To estimate $I$, let $y$ be any point in $B_{o}(R) \backslash B_{o}(2 r)$, then $r_{1}=r(y) \geq 2 r=2 r(x)$ and $r(z, y) \geq \frac{1}{2} r_{1}$ if $z \in B_{o}(r)$. Also $B_{o}\left(\frac{1}{2}\right) \subseteq B_{o}(2 r)$. Hence by the subgradient estimate [CKT],

$$
\begin{aligned}
\left|G_{R}(o, y)-G_{R}(x, y)\right| & \leq r \sup _{z \in B_{o}(r)}\left|\nabla_{b, z} G_{R}(z, y)\right| \\
& \leq C_{1} \frac{r}{r_{1}} \sup _{z \in B_{o}(r)} G_{R}(z, y) \\
& \leq C_{2} \frac{r}{r_{1}} G(o, y) \\
& \leq C_{3} \frac{r}{r_{1}} \int_{r_{1}}^{\infty} \frac{t}{V\left(b_{o}(t)\right)} d t
\end{aligned}
$$


where $C_{1}, C_{2}, C_{3}$ are constant depending only on $n$. Here we used the Harnack inequality for $G_{R}(., y)$ and the fact that $G_{R}(o, y) \leq G(o, y)$.

$$
\begin{aligned}
|I| \leq & C_{3} r \int_{B_{o}(R) \backslash B_{o}(2 r)} \frac{1}{r(y)}\left(\int_{r(y)}^{\infty} \frac{t}{V\left(B_{o}(t)\right)} d t\right) f(y) d y \\
= & C_{3} r \int_{2 r}^{R} t^{-1}\left(\int_{t}^{\infty} \frac{t}{V\left(B_{o}(t)\right)} d t\right)\left(\int_{\partial B_{o}(t)} f(y) d y\right) d t \\
= & C_{3} r\left[\left.t^{-1}\left(\int_{t}^{\infty} \frac{t}{V\left(B_{o}(t)\right)} d t\right)\left(\int_{B_{o}(t)} f(y) d y\right)\right|_{2 r} ^{R}\right. \\
& \left.-\int_{2 r}^{R}\left(\int_{B_{o}(t)} f(y) d y\right)\left[-\frac{1}{t^{2}} \int_{t}^{\infty} \frac{s}{V\left(B_{o}(s)\right)} d s-\frac{1}{V\left(B_{o}(t)\right)}\right] d t\right] \\
\leq & C_{3} r\left[R^{-1}\left(\int_{R}^{\infty} \frac{R}{V\left(B_{o}(R)\right)} d t\right)\left(\int_{B_{o}(R)} f(y) d y\right)\right. \\
& \left.+\int_{2 r}^{R}\left(\int_{B_{o}(t)} f(y) d y\right)\left[\frac{1}{t^{2}} \int_{t}^{\infty} \frac{s}{V\left(B_{o}(s)\right)} d s+\frac{1}{V\left(B_{o}(t)\right)}\right] d t\right] \\
\leq & C_{4} r\left[R k(R)+\int_{2 r}^{R} k(t) d t\right]
\end{aligned}
$$

for some positive constants $C_{4}$ which depend on $n$ and $\sigma$. Here we have used (2.14). Moreover

$$
\begin{aligned}
\int_{B_{o}(2 r)} G_{R}(o, y) f(y) d y \leq & \int_{B_{o}(2 r)} G(o, y) f(y) d y \\
\leq & C_{5} \int_{0}^{2 r}\left(\int_{t}^{\infty} \frac{s}{V\left(B_{o}(s)\right)} d s\right)\left(\int_{\partial B_{o}(t)} f\right) d t \\
= & C_{5}\left[\left.\left(\int_{t}^{\infty} \frac{s}{V\left(B_{o}(s)\right)} d s\right)\left(\int_{B_{o}(t)} f\right)\right|_{0} ^{2 r}\right. \\
& \left.+\int_{0}^{2 r} \frac{t}{V\left(B_{o}(t)\right)}\left(\int_{B_{o}(t)} f(y)\right) d t\right] \\
\leq & C_{5}\left[\left(\int_{2 r}^{\infty} \frac{s}{V\left(B_{o}(s)\right)} d s\right)\left(\int_{B_{o}(2 r)} f\right)\right. \\
& \left.+\int_{0}^{2 r} \frac{t}{V\left(B_{o}(t)\right)}\left(\int_{B_{o}(t)} f(y)\right) d t\right] \\
\leq & C_{5}\left[C_{6} r^{2} k(2 r)+\int_{0}^{2 r} t k(t) d t\right]
\end{aligned}
$$

for some positive constants $C_{5}, C_{6}$ which depend on $n$ and $\sigma$. From (2.15), (2.17) and (2.18), we have

$$
u_{R}(x) \leq C_{7}\left(r R k(R)+r^{2} k(2 r)+r \int_{2 r}^{R} k(t) d t\right)+\beta_{1} \int_{0}^{2 r} t k(t) d t
$$

for some positive constants $C_{7}, \beta_{1}$ which depend on $n$ and $\sigma$. As in the proof of (2.18), using the lower bound of the Green's function, we have

$$
\int_{B_{o}(2 r)} G(o, y) f(y) d y \geq C_{8}\left[C_{9} r^{2} k(2 r)+\int_{0}^{2 r} t k(t) d t\right]
$$

for some positive constants $C_{8}, C_{9}$ which depend on $n$ and $\sigma$. For $1>\varepsilon>0$,

$$
\begin{aligned}
\int_{B_{x}(\varepsilon r)} G_{R}(x, y) f(y) d y \leq & \int_{B_{x}(\varepsilon r)} G(x, y) f(y) d y \\
\leq & \beta_{2}\left[\int_{\varepsilon r}^{\infty} \frac{t}{V\left(B_{x}(t)\right)} d t\left(\int_{B_{x}(\varepsilon r)} f d y\right)\right. \\
& \left.+\int_{0}^{\varepsilon r} \frac{t}{V\left(B_{x}(t)\right)}\left(\int_{B_{x}(t)} f d y\right) d t\right] \\
\leq & C_{10}(\varepsilon r)^{2} k(x, \varepsilon r)+\beta_{2} \int_{0}^{\varepsilon r} t k(x, t) d t \\
\leq & C_{11} r^{2} k((1+\varepsilon) r)+\beta_{2} \int_{0}^{\varepsilon r} t k(x, t) d t
\end{aligned}
$$


for some positive constants $C_{10}, \beta_{2}$ which depend on $n$ and $\sigma$ and $C_{11}$ which depends on $n, \varepsilon$ and $\sigma$. Here we used volume doubling property and the fact that $B_{x}(\varepsilon r) \subset B_{o}((1+\varepsilon) r)$.

$$
\begin{aligned}
\int_{B_{o}(2 r) \backslash B_{x}(\varepsilon r)} G_{R}(x, y) f(y) d y & \leq \int_{B_{o}(2 r) \backslash B_{x}(\varepsilon r)} G(x, y) f(y) d y \\
& \leq \sigma \frac{16 r^{2}}{V_{x}(\varepsilon r)} \int_{B_{o}(2 r)} f(y) d y \\
& \leq C_{12} r^{2} k(2 r)
\end{aligned}
$$

for some positive constant $C_{12}$ which depends on $n, \varepsilon$ and $\sigma$. From (2.15), (2.17), (2.20), (2.21) and (2.22), if $R \geq 4 r$, we have

$$
u_{R}(x) \geq-C_{13} r\left(R k(R)+\int_{2 r}^{R} k(t) d t\right)-\beta_{2} \int_{0}^{\varepsilon r} t k(x, t) d t+\beta_{3} \int_{0}^{2 r} t k(t) d t
$$

for some positive constant $C_{13}$ which depend on $n, \varepsilon$ and $\sigma$ and $\beta_{3}$ depends on $n$. Here we have used the fact that for any $\alpha>1, k(\alpha R) \geq C k(R)$ for some positive constant $C$ which depends on $n, \alpha$ for all $R$.

Since $\int_{0}^{\infty} k(t) d t<\infty, \lim _{R \rightarrow \infty} R k(R)=0$. Hence from (2.19) and (2.23), $u_{R}(x)$ is bounded on compacts sets and there exists $R_{i} \rightarrow \infty$ such that $u_{R_{i}}$ converges uniformly on compact sets to a function $u$ which satisfies $\triangle_{b} u=f$. By (2.19) and (2.23), let $R_{i} \rightarrow \infty$ we can conclude that $u$ satisfies the estimates in the theorem.

In order to prove Theorem 1.1 we need one more lemma,

Lemma 2.1. ([NST) Let $M=M_{1} \times M_{2}$, where $M_{1}$ and $M_{2}$ are complete noncompact manifolds with nonnegative Ricci curvature. Let $f \geq 0$ be a function on $M_{1}$ and be considered also as a function on $M$, which is independent of the second variable. Let $x=\left(x_{1}, x_{2}\right) \in M$ and $r>0$. Then

$$
\frac{C^{-1}}{V\left(B_{x_{1}}^{(1)}\left(\frac{1}{\sqrt{2}} r\right)\right)} \int_{B_{x_{1}}^{(1)}\left(\frac{1}{\sqrt{2}} r\right)} f \leq \frac{1}{V\left(B_{x}(r)\right)} \int_{B_{x}(r)} f \leq \frac{C}{V\left(B_{x_{1}}^{(1)}(r)\right)} \int_{B_{x_{1}}^{(1)}(r)} f
$$

for some constant $C>0$ depending only on the dimension of $M_{1}$ and $M_{2}$. Here $B_{x}(t), B_{x_{1}}^{(1)}(t)$ are geodesic balls with radius $t$ in $M, M_{1}$ with centers at $x, x_{1}$ respectively.

The proof of Theorem 1.1 :

Proof. Let $\widetilde{M}=M^{2 n+1} \times \mathbb{R}^{4}$ with the standard Euclidean space $\mathbb{R}^{4}$. Then $\widetilde{M}$ is nonparabolic. By volume doubling property, we will prove that $(2.14)$ is true on $\widetilde{M}$. It follows from [CCHT, Corollary 1.1.] again that

$$
V\left(B_{x}(t)\right)=V\left(B_{x}\left(\frac{t}{r(x, y)} r(x, y)\right)\right) \leq C_{v}\left(\frac{t}{r(x, y)}\right)^{2 C} V\left(B_{x}(r(x, y))\right)
$$


where $C_{v}$ is a positive constant which depends only on $n$, so we have

$$
V^{-1}\left(B_{x}(r(x, y))\right) C_{v}^{-1}\left(\frac{t}{r(x, y)}\right)^{-2 C} \leq V^{-1}\left(B_{x}(t)\right) .
$$

That is

$$
\frac{t}{V\left(B_{x}(r(x, y))\right)} C_{v}^{-1}\left(\frac{t}{r(x, y)}\right)^{-2 C} \leq \frac{t}{V\left(B_{x}(t)\right)} .
$$

On the other hand, we will have

$$
\frac{t}{V\left(B_{x}(t)\right)} \leq C_{v}\left(\frac{t}{r(x, y)}\right)^{-2 C} \frac{t}{V\left(B_{x}(r(x, y))\right)} .
$$

From (2.26) and (2.27), we have

$$
\frac{t}{V\left(B_{x}(r(x, y))\right)} C_{v}^{-1}\left(\frac{t}{r(x, y)}\right)^{-2 C} \leq \frac{t}{V\left(B_{x}(t)\right)} \leq C_{v}\left(\frac{t}{r(x, y)}\right)^{-2 C} \frac{t}{V\left(B_{x}(r(x, y))\right)}
$$

and then

$$
C_{1}^{-1} \frac{r^{2}(x, y)}{V\left(B_{x}(r(x, y))\right)} \leq \int_{r(x, y)}^{\infty} \frac{t}{V\left(B_{x}(t)\right)} d t \leq C_{1} \frac{r^{2}(x, y)}{V\left(B_{x}(r(x, y))\right)}
$$

where $C_{1}$ is a positive constant depending only on $n$. From (2.1), we have

$$
\sigma^{-1} \frac{r^{2}(x, y)}{V\left(B_{x}(r(x, y))\right)} \leq G(x, y) \leq \sigma \frac{r^{2}(x, y)}{V\left(B_{x}(r(x, y))\right)},
$$

where $\sigma$ is a positive constant depending only on $n$. So we know that (2.12) is satisfied on $\widetilde{M}$. Then the rest proof is similar to the proof as in [NST, Theorem 1.2.], so we omit it here.

Corollary 2.1. Let $(M, J, \theta)$ be a complete noncompact strictly pseudoconvex $C R(2 n+1)$-manifold with nonnegative pseudohermitian Ricci curvature tensors and vanishing torsion. Let $f$ be a nonnegative locally Hölder continuous function. Assume that $u$ is a solution of the CR Poisson equation

$$
\triangle_{b} u=f
$$

If $f_{0}=0$, then

$$
u_{0}=0 .
$$

Proof. It follows from CKL that $\left[\Delta_{b}, \mathbf{T}\right] u=0$ if the torsion is vanishing that

$$
\triangle_{b} u_{0}=f_{0} .
$$

Then from Theorem 1.1

$$
\begin{aligned}
\alpha_{1} r \int_{2 r}^{\infty} \widetilde{k}(t) d t+\beta_{1} \int_{0}^{2 r} t \widetilde{k}(t) d t & \geq u(x) \\
& \geq-\alpha_{2} r \int_{2 r}^{\infty} \widetilde{k}(t) d t-\beta_{2} \int_{0}^{\varepsilon r} t \widetilde{k}(x, t) d t+\beta_{3} \int_{0}^{2 r} t \widetilde{k}(t) d t
\end{aligned}
$$


where $\widetilde{k}(x, t)=\frac{1}{V_{x}(t)} \int_{B_{x}(t)} f_{0} d \mu$, and $\widetilde{k}(t)=\widetilde{k}(o, t)$. If $f_{0}=0$, we can obtain that $u_{0}=0$ as well.

Observe that if $\int_{0}^{\infty} k(o, t) d t<\infty$, then $\int_{0}^{\infty} k(x, t) d t<\infty$ for all $x$. If $u$ is the solution obtained as in Theorem 1.1, then for any $x_{0} \in M$

$$
u(x)-u\left(x_{0}\right)=\int_{M}\left(G\left(x_{0}, y\right)-G(x, y)\right) f(y) d y .
$$

From the proof of the theorem, it is easy to see that

$$
\begin{aligned}
\alpha_{1} r \int_{2 r}^{\infty} k\left(x_{0}, t\right) d t+\beta_{1} \int_{0}^{2 r} t k\left(x_{0}, t\right) d t & \geq u(x)-u\left(x_{0}\right) \\
& \geq-\alpha_{2} r \int_{2 r}^{\infty} k\left(x_{0}, t\right) d t-\beta_{2} \int_{0}^{\varepsilon r} t k(x, t) d t+\beta_{3} \int_{0}^{2 r} t k\left(x_{0}, t\right) d t,
\end{aligned}
$$

where $\alpha_{i}$ and $\beta_{j}$ are the constants in Theorem 1.1 and $r=r\left(x, x_{0}\right)$.

From (2.30), we can obtain the following results.

Theorem 2.3. The same assumptions and notation as in Theorem 1.1 or in Theorem 2.2, Let $u$ be the solution of

$$
\triangle_{b} u=f
$$

obtained as in Theorem 1.1 or in Theorem 2.2, Then

(i)

$$
\left|\nabla_{b} u(x)\right| \leq C(n, \sigma) \int_{0}^{\infty} k(x, t) d t
$$

where $C$ is a positive constant depending only on $n$.

(ii) For any $p \geq 1$ and $\alpha \geq 2$, if $u$ is a solution obtained in Theorem 2.2, then

$$
\frac{1}{V_{o}(R)} \int_{B_{o}(R)}\left|\nabla_{b} u\right|^{p} d x \leq C(n, p)\left(\int_{\alpha R}^{\infty} k(t) d t\right)^{p}+\frac{C(n, p, \alpha) R^{p}}{V\left(B_{o}(R)\right)} \int_{B_{o}(\alpha R)} f^{p} d x
$$

and if $u$ is a solution obtained in Theorem 1.1, then

$$
\frac{1}{V_{o}\left(\frac{1}{\sqrt{2}} R\right)} \int_{B_{o}\left(\frac{1}{\sqrt{2}} R\right)}\left|\nabla_{b} u\right|^{p} d x \leq C(n, p)\left(\int_{\alpha R}^{\infty} k(t) d t\right)^{p}+\frac{C(n, p, \alpha) R^{p}}{V\left(B_{o}(R)\right)} \int_{B_{o}(\alpha R)} f^{p} d x .
$$

(iii) If $f_{0}=0$ and $u$ is a solution obtained in Theorem 2.2, then

$$
\frac{1}{V_{o}(R)} \int_{B_{o}(R)}\left|\left(\nabla^{H}\right)^{2} u\right|^{2} \leq C(n)\left[R^{-2}\left(\int_{4 R} k(t) d t\right)^{2}+\frac{1}{V_{o}(4 R)} \int_{B_{o}(4 R)} f^{2}\right]
$$

and if $u$ is a solution obtained in Theorem 1.1, then

$$
\frac{1}{V_{o}\left(\frac{1}{\sqrt{2}} R\right)} \int_{B_{o}\left(\frac{1}{\sqrt{2}} R\right)}\left|\left(\nabla^{H}\right)^{2} u\right|^{2} \leq C(n, \sigma)\left[R^{-2}\left(\int_{4 R} k(t) d t\right)^{2}+\frac{1}{V_{o}(4 R)} \int_{B_{o}(4 R)} f^{2}\right] .
$$


Proof. Let us first consider the solution $u$ obtained in Theorem 2.2 .

(i). From (2.30), we can know that (i) is true.

(ii) From Theorem 2.2] for any $x \in B_{o}(R)$ and (2.12) and the subgradient estimate [CKT], we have

$$
\begin{aligned}
\left|\nabla_{b} u\right| \leq & \int_{M}\left|\nabla_{b, x} G(x, y)\right| f(y) d y \\
\leq & C_{1}(n) \int_{M} r^{-1}(x, y) G(x, y) f(y) d y \\
\leq & C_{2}(n, \sigma) \int_{M \backslash B_{o}(\alpha R)} \frac{r(x, y)}{V\left(B_{x}(r(x, y))\right)} f(y) d y \\
& +C_{1}(n) \int_{B_{x}(\alpha R)} r^{-1}(x, y) G(x, y) f(y) d y
\end{aligned}
$$

for some positive constants $C_{1}(n)$ and $C_{2}(n, \sigma)$.

$$
\begin{aligned}
& \int_{M \backslash B_{o}(\alpha R)} \frac{r(x, y)}{V\left(B_{x}(r(x, y))\right)} f(y) d y \\
& \leq C_{3}(n) \int_{M \backslash B_{o}(\alpha R)} \frac{r(y)}{V\left(B_{o}(r(y))\right)} f(y) d y \\
& =\int_{\alpha R}^{\infty} \frac{t}{V_{o}(t)}\left(\int_{\partial B_{o}(r)} f\right) d t \\
& =C_{3}(n)\left[\left.\frac{t}{V_{o}(t)} \int_{B_{o}(t)} f\right|_{\alpha R} ^{\infty}-\int_{\alpha R}^{\infty}\left(\int_{B_{o}(t)} f\right) \frac{d}{d t}\left(\frac{t}{V_{o}(t)}\right) d t\right] \\
& \leq C_{3}(n)\left[-\int_{\alpha R}^{\infty}\left(\frac{1}{V_{o}(t)}-\frac{t A_{o}(t)}{V_{o}(t)}\right)\left(\int_{B_{o}(t)} f\right) d t\right] \\
& \leq C_{4}(n) \int_{\alpha R}^{\infty} \frac{1}{V_{o}(t)}\left(\int_{B_{o}(t)} f\right) d t=C_{4}(n) \int_{\alpha R}^{\infty} k(t) d t
\end{aligned}
$$

for some constants $C_{3}(n), C_{4}(n)$, where we have used the fact that $\alpha \geq 2, t k(t) \rightarrow 0$ as $t \rightarrow \infty$ and CCHT] Corollary 1.1.] $t A_{o}(t) \leq C_{5}(n)(n+3) V_{o}(t)$, where $C_{5}(n)$ is a positive constant. Note that for any $z \in M$ and for $\rho>0$, we have

$$
\int_{B_{z}(\rho)} \frac{r(z, y)}{V_{z}(r(z, y))} d y=\int_{0}^{\rho} \frac{t A_{z}(t)}{V_{z}(t)} d t \leq C_{5}(n)(n+3) \rho .
$$

Let us first assume $p>1$ and let $q=\frac{p}{p-1}$. From (2.31) and (2.32), we have

$$
\begin{aligned}
\int_{B_{o}(R)}\left|\nabla_{b} u\right|^{p} \leq & C_{6}(n, \sigma, p) V_{o}(R)\left(\int_{\alpha R}^{\infty} k(t) d t\right)^{p} \\
& +C_{7}(n, p) \int_{B_{o}(R)}\left(\int_{B_{o}(\alpha R)} r^{-1}(x, y) G(x, y) f(y) d y\right)^{p} d x
\end{aligned}
$$

for some constants $C_{6}(n), C_{7}(n)$. From Theorem 2.2 for any $x \in B_{o}(R)$ and (2.12) and (2.33), we have

$$
\begin{aligned}
& \int_{B_{o}(R)}\left(\int_{B_{\alpha R}} r^{-1}(x, y) G(x, y) f(y) d y\right)^{p} d x \\
& \leq q \int_{B_{o}(R)}\left(\int_{B_{o}(\alpha R)} r^{-1}(x, y) G(x, y) d y\right)^{\frac{p}{q}}\left(i n t_{B_{o}(\alpha R)} r^{-1}(x, y) G(x, y) f^{p}(y) d y\right) d x \\
& \leq \int_{B_{o}(R)}\left(\int_{B_{o}(\alpha R)} \frac{\sigma r(x, y)}{V_{x}(r(x, y))} d y\right)^{\frac{p}{q}}\left(\int_{B_{o}(\alpha R)} r^{-1}(x, y) G(x, y) f^{p}(y) d y\right) d x \\
& \leq C_{8}(n, \sigma, p, \alpha) R^{\frac{p}{q}} \int_{B_{o}(R)}\left(\int_{B_{o}(\alpha R)} r^{-1}(x, y) G(x, y) f^{p}(y) d y\right) d x \\
& =C_{8}(n, \sigma, p, \alpha) R^{\frac{p}{q}} \int_{B_{o}(\alpha R)}\left(\int_{B_{o}(R)} r^{-1}(x, y) G(x, y) d x\right) f^{p}(y) d y \\
& \leq C_{9}(n, \sigma, p, \alpha) R^{p} \int_{B_{0}(\alpha R)} f^{p}(y) d y
\end{aligned}
$$


for some constants $C_{8}(n, \sigma, p, \alpha)$ and $C_{9}(n, \sigma, p, \alpha)$. Combine this with (2.34), (ii) follows if $p>1$. The case $p=1$ can be proved similarly.

(iii) By the assumption $f_{0}=0$, Corollary 2.1 and (2.35), we have $u_{0}=0$ and

$$
\begin{aligned}
\frac{1}{2} \triangle_{b}\left|\nabla_{b} u\right|^{2} & =\left|\left(\nabla^{H}\right)^{2} u\right|^{2}+\left\langle\nabla_{u}, \nabla_{b} \triangle_{b} u\right\rangle+2 \operatorname{Ric}\left(\left(\nabla_{b} u\right)_{C},\left(\nabla_{b} u\right)_{C}\right) \\
& \geq\left|\left(\nabla^{H}\right)^{2} u\right|^{2}+\left\langle\nabla_{b} u, \nabla_{b} \triangle_{b} u\right\rangle=\left|\left(\nabla^{H}\right)^{2} u\right|^{2}+\left\langle\nabla_{b} u, \nabla_{b} f\right\rangle,
\end{aligned}
$$

where we have used the Ric $\geq 0$. Let $\eta \geq 0$ be a smooth function with compact in $B_{o}(2 R)$. Multiplying (2.36) by $\eta^{2}$ and integrating by parts, we have

$$
\begin{aligned}
& \int_{B_{o}(2 R)} \eta^{2}\left|\left(\nabla^{H}\right)^{2} u\right|^{2} \leq \int_{B_{o}(2 R)}\left[\frac{1}{2} \eta^{2} \triangle_{b}\left|\nabla_{b} u\right|^{2}-\eta^{2}\left\langle\nabla_{b} u, \nabla_{b} f\right\rangle\right] \\
& \leq \int_{B_{o}(2 R)} \eta^{2} f^{2}+\int_{B_{o}(2 R)} \eta\left|\nabla_{b} \eta\right|\left|\nabla_{b} u\right||f|+\left.2 \int_{B_{o}(2 R)} \eta\left|\nabla_{b} \eta\right|\left|\nabla_{b}\right| \nabla_{b} u\right|^{2} \mid \\
& \leq C\left[\int_{B_{o}(2 R)} \eta^{2} f^{2}+\int_{B_{o}(2 R)}\left|\nabla_{b} \eta\right|^{2}\left|\nabla_{b} u\right|^{2}+\int_{B_{o}(2 R)} \eta\left|\nabla_{b} \eta\right|\left|\nabla_{b} u \|\left(\nabla^{H}\right)^{2} u\right|\right] \\
& \leq C\left[\int_{B_{o}(2 R)} \eta^{2} f^{2}+\left(1+\frac{1}{\varepsilon}\right) \int_{B_{o}(2 R)}\left|\nabla_{b} \eta\right|^{2}\left|\nabla_{b} u\right|^{2}+\varepsilon \int_{B_{o}(2 R)} \eta^{2}\left|\left(\nabla^{H}\right)^{2} u\right|^{2}\right]
\end{aligned}
$$

for any $\varepsilon>0$, some absolute constant $C$. Choose $\varepsilon=\frac{1}{2 C}$, we have

$$
\int_{B_{o}(R)}\left|\left(\nabla^{H}\right)^{2} u\right|^{2} \leq \widetilde{C}\left[\int_{B_{o}(2 R)} f^{2}+R^{-2} \int_{B_{o}(2 R)}\left|\nabla_{b} u\right|^{2}\right]
$$

for all $\eta \geq 0$ with compact support in $B_{o}(R)$. Choose a suitable $\eta$, we have

$$
\int_{B_{o}(2 R)} \eta^{2}\left|\left(\nabla^{H}\right)^{2} u\right|^{2} \leq 2 C\left[\int_{B_{o}(2 R)} \eta^{2} f^{2}+\int_{B_{o}(2 R)}\left|\nabla_{b} \eta\right|^{2}\left|\nabla_{b} u\right|^{2}\right]
$$

for some absolute constant $\widetilde{C}$. Combining this with (ii) the results follows.

Suppose the assumption of Theorem 1.1 are satisfied. By the proof Theorem 1.1. Lemma 2.1 and the above proof, we can obtain the other results are also true.

\section{The CR Heat Equation}

In this section, we will derive some basic results for solutions to the $\mathrm{CR}$ heat equation on complete noncompact pseudohermitian $(2 n+1)$-manifolds. We refer to $\overline{\mathrm{CF}}$, $\mathrm{CHL}$, $\mathrm{CCHT}$, BBG for some basic properties.

We first recall a basic theorem for solutions to the CR heat equation from [CHL] and [NT1].

Theorem 3.1. Let $(M, J, \theta)$ be a complete noncompact strictly pseudoconvex $C R(2 n+1)$-manifold with nonnegative pseudohermitian Ricci curvature tensors and vanishing torsion. Let $u$ be a continuous function on $M$ such that

$$
\frac{1}{V\left(B_{o}(r)\right)} \int_{B_{o}(r)}|u|(x) d x \leq \exp \left(a r^{2}+b\right)
$$


for some positive constant $a>0$ and $b>0$. Then the following initial value problem

$$
\left\{\begin{array}{l}
\left(\frac{\partial}{\partial t}-\triangle_{b}\right) v(x, t)=0, \\
v(x, 0)=u(x)
\end{array}\right.
$$

has a solution on $M \times\left(0, \frac{C}{16 a}\right]$. Moreover,

$$
v(x, t)=\int_{M} H(x, y, t) u(y) d y
$$

where $H(x, y, t)$ is the $C R$ heat kernel of $M$.

For later proposes, we need more basic results from Theorem 3.1] and [NT2].

Lemma 3.1. Let $(M, J, \theta)$ be a complete noncompact strictly pseudoconvex $C R(2 n+1)$-manifold with nonnegative pseudohermitian Ricci curvature tensors and vanishing torsion. Let $u, v$ be as in Theorem 3.1. Then for any $0<\varepsilon<1$, there exists a constant $C=C(n, \varepsilon, a, b)$ and $0<T_{0}<\frac{C(\varepsilon)}{16 a}$ such that for all $(x, t) \in M \times\left(0, T_{0}\right]$ with $r^{2}(x) \geq T_{0}$,

$$
\left|v(x, t)-\int_{B_{x}(\varepsilon r)} H(x, y, t) u(y)\right| \leq C(n, \varepsilon, a, b),
$$

where $r=r(x)$.

Corollary 3.1. With the same assumption and notations as in Lemma 3.1. Then for $x \in M$ with $r=$ $r(x) \geq \sqrt{T_{0}}$ such that $u \geq 0$ on $B_{x}(\varepsilon r)$, then for any $0 \leq t<T_{0}$

$$
-C(n, \varepsilon, a, b)+C(n, \varepsilon) \inf _{B_{x}(\varepsilon r)} u \leq v(x, t) \leq C(n, \varepsilon, a, b)+\sup _{B_{x}(\varepsilon r)} u
$$

for some positive constant $C(n, \varepsilon)$.

Suppose that $|u(x)-u(y)| \leq \beta r(x, y)$, then, by Theorem 3.1, $v$ is defined for all $t$. We have the following.

Lemma 3.2. Let $(M, J, \theta)$ be a complete noncompact strictly pseudoconvex $C R(2 n+1)$-manifold with nonnegative pseudohermitian Ricci curvature tensors and vanishing torsion. Suppose that $u_{0}(x)=0$ and $|u(x)-u(y)| \leq \beta r(x, y)$ for all $x, y \in M$ and let $v$ be the solution of the heat equation with initial value $u$ obtained in Theorem 3.1. Then for all $t>0$,

$$
\sup _{x \in M}\left|\nabla_{b} v(x, t)\right| \leq \beta .
$$


Lemma 3.3. Let $(M, J, \theta)$ be a complete noncompact strictly pseudoconvex $C R(2 n+1)$-manifold with nonnegative pseudohermitian Ricci curvature tensors and vanishing torsion. Let $u$ be a smooth function on $M$ with bounded gradient and let $v$ be the solution of the heat equation initial value $u$ as in (3.1) with $u_{0}(x)=0$. Then for any $t>0$

$$
\sup _{M}\left|\nabla_{b} v(., t)\right| \leq \sup _{M}\left|\nabla_{b} u\right| .
$$

\section{Poincaré-Lelong Equation}

In this section, assume that $\rho$ is a given real closed $(1,1)$-form on $M$ with $\rho_{\alpha \bar{\beta}, 0}=0$. We will solve the CR Poincare- Lelong equation

$$
i \partial_{b} \bar{\partial}_{b} u=\rho
$$

by first solving the CR Poisson equation

$$
\Delta_{b} u=2 f
$$

for $f=\operatorname{trace}(\rho)$. In fact, by commutation relation (A.2), the CR Poincare- Lelong equation (4.1) implies

$$
\triangle_{b} u+h u_{0}=2 f,
$$

where $h=\sum_{\alpha=1}^{n} h_{\alpha \bar{\alpha}}$. In the present paper, it follows from Corollary 2.1 that $u_{0}(x)=0$ and then 4.3 reduces to the CR Poisson equation (4.2).

Note that it follows from Theorem 1.1 that one can solve the CR Poisson equation $\triangle_{b} u=2 f$ with $u$ satisfying (1.7). Moreover, $u$ is given by

$$
u(x)=2 \int_{M}(G(o, y)-G(x, y)) f(y) d y .
$$

Theorem 1.2 will follow from Lemma 4.1 and Lemma 4.2 below.

Lemma 4.1. The same assumption as in Theorem 1.2. Then

(i) The Cauchy problem (3.1) with initial value u has long time solution $v(x, t)$ which is given by

$$
v(x, t)=\int_{M} H(x, y, t) u(y) d y
$$

and

$$
v_{0}(x, t)=0 .
$$

(ii) There exists $t_{i} \rightarrow \infty$ such that $v\left(x, t_{i}\right)-v\left(o, t_{i}\right)$ together with their derivatives converge uniformly on compact subsets to a constant function. 
Proof. (i) We want to apply Theorem 3.1 For any $R>0$ and $x \in B_{o}(R)$,

$$
\begin{aligned}
|u(x)| \leq & 2 \int_{M}|G(o, y)-G(x, y)| f(y) d y \\
\leq & 2 \int_{M \backslash B_{o}(4 r)}|G(o, y)-G(x, y)| f(y) d y \\
& +2 \int_{B_{o}(4 R)}|G(o, y)-G(x, y)| f(y) d y \\
= & I(x)+I I(x) .
\end{aligned}
$$

It follow from the proof of (2.17) and the condition (1.5), we have

$$
I(x) \leq C(n) r(x) \int_{4 R}^{\infty} k(s) d s \leq C(n) r(x),
$$

where $C(n)$ is a positive constant. On the other hand, we have

$$
\begin{aligned}
\int_{B_{o}(R)} I I(x) d x & \leq \int_{x \in B_{o}(R)}\left[2 \int_{y \in B_{o}(4 R)}(G(o, y)+G(x, y)) f(y) d y\right] d x \\
& =\int_{y \in B_{o}(4 R)}\left[2 \int_{B_{o}(R)}(G(o, y)+G(x, y)) d x\right] f(y) d y
\end{aligned}
$$

and from the proof of (2.18),

$$
\begin{aligned}
& \int_{y \in B_{o}(4 R)}\left[\int_{B_{o}(R)} G(o, y) d x\right] f(y) d y \\
= & 2 V_{o}(R) \int_{B_{o}(4 R)} G(o, y) f(y) d y \\
\leq & C(n) V_{o}(R)\left[R^{2} k(4 R)+\int_{0}^{4 R} t k(t) d t\right] .
\end{aligned}
$$

Moreover from [CCHT, Corollary 1.1.], we have

$$
t A_{o}(t) \leq C(n) V\left(B_{o}(t)\right)
$$

Thus

$$
\begin{aligned}
& 2 \int_{y \in B_{o}(4 R)}\left[\int_{x \in B_{o}(R)} G(x, y) d x\right] f(y) d y \\
& \leq C(n) \int_{y \in B_{o}(4 R)}\left[\int_{x \in B_{o}(R)} \frac{r^{2}(x, y)}{V\left(B_{y}(r(x, y))\right.} d x\right] f(y) d y \\
& \leq C(n) \int_{y \in B_{o}(4 R)}\left[\int_{x \in B_{y}(5 R)} \frac{r^{2}(x, y)}{V\left(B_{y}(r(x, y))\right.} d x\right] f(y) d y \\
& =C(n) \int_{y \in B_{o}(4 R)}\left[\int_{0}^{5 R} \frac{t^{2} A_{y}(t)}{V\left(B_{y}(t)\right)} d t\right] f(y) d y \\
& \leq C(n) V\left(B_{o}(R)\right) R^{2} k(4 R) .
\end{aligned}
$$

All together with (4.7), (4.7) and (4.8), we have

$$
\int_{B_{o}(R)} I I(x) d x \leq C(n) V\left(B_{o}(R)\right)\left(R^{2} k(4 R)+\int_{0}^{4 R} s k(s) d s\right) .
$$


Also from (4.5), (4.6), (4.9) and (1.5), we have

$$
\frac{1}{V\left(B_{o}(R)\right)} \int_{B_{o}(R)}|u| \leq C(n)\left(1+R^{2}\right) .
$$

Then (i) of Lemma follows easily from Theorem 3.1. Furthermore, since the torsion is vanishing, it is easy to know that $v_{0}(x, t)$ is a solution of the following system:

$$
\left\{\begin{array}{l}
\left(\frac{\partial}{\partial t}-\triangle_{b}\right) \mu(x, t)=0, \\
\mu(x, 0)=0 .
\end{array}\right.
$$

It follows from Theorem 3.1 again that

$$
v_{0}(x, t)=\mathbf{T}(v(x, t))=0
$$

on $M \times[0, \infty)$.

(ii) Let us first give an estimate of $\left|\nabla_{b} v\right|$. We proceed as in proof of Theorem 3.1 Namely, use cut-off functions $\varphi_{i}$ and denote $f_{i}=\varphi_{i} f$. Solve

$$
\triangle_{b} u=2 f_{i}
$$

by using (4.4) and find the solution $v_{i}$ of (3.1) with initial value $u_{i}$. Then $v_{i}$ subconverge to $v$ together with their derivatives uniformly on compact sets on $M \times[0, \infty)$. From Theorem 2.3, we know that $\left|\nabla_{b} u_{i}\right|$ is bounded, and hence $\left|\nabla_{b} v_{i}\right|$ is bounded by Lemma 3.3. We can apply the maximum principle to $\left|\nabla_{b} v_{i}\right|$ which is a subsolution of the CR heat equation and conclude that for any $x$ such that $r(x) \leq \sqrt{t}$,

$$
\begin{aligned}
\left|\nabla_{b} v_{i}\right| & \leq \int_{M} H(x, y, t)\left|\nabla_{b} u_{i}\right| d y \\
& \leq C(n) \sup _{r \geq \sqrt{t}} \frac{1}{V\left(B_{x}(r)\right)} \int_{B_{x}(r)}\left|\nabla_{b} u_{i}\right|(y) d y \\
& \leq C(n) \sup _{r \geq \sqrt{t}} \frac{1}{V\left(B_{o}(2 r)\right)} \int_{B_{o}(2 r)}\left|\nabla_{b} u_{i}\right|(y) d y \\
& \leq C(n) \sup _{r \geq \sqrt{t}}\left(\int_{4 r}^{\infty} k(s) d s+r k(4 r)\right) \\
& \leq C(n) \int_{4 \sqrt{t}}^{\infty} k(s) d s
\end{aligned}
$$

for some positive constant $C(n)$. Here we have used [CF, (3.13)] and Theorem 2.3 and CR Volume doubling property as well as the fact that $0 \leq f_{i} \leq f$. Hence

$$
\sup _{x \in B_{o}(\sqrt{t})}\left|\nabla_{b} v_{i}\right|(x, t) \leq C(n) \int_{4 \sqrt{t}}^{\infty} k(s) d s
$$


for all $i$ and then

$$
\sup _{x \in B_{o}(\sqrt{t})}\left|\nabla_{b} v\right|(x, t) \leq C(n) \int_{4 \sqrt{t}}^{\infty} k(s) d s .
$$

On the other hand, $f_{i}$ has compact support, $u_{i}$ and $v_{i}$ are bounded. Since $\left(v_{i}\right)_{t}$ is a solution of the following system:

$$
\left\{\begin{array}{l}
\left(\frac{\partial}{\partial t}-\triangle_{b}\right) \mu(x, t)=0 \\
\mu(x, 0)=2 f_{i}(x)
\end{array}\right.
$$

as in the proof of Lemma 3.3. one can prove that for any $T>0$, there exist constant $C_{i}(n)$ such that

$$
\int_{0}^{T}\left[\frac{1}{V\left(B_{o}(r)\right)} \int_{B_{o}(r)}\left|\left(v_{i}\right)_{t}\right|^{2} d y\right] d t \leq C_{i}(n)
$$

for all $r$. Hence we can apply maximum principle and conclude that

$$
\begin{aligned}
\frac{\partial v_{i}}{\partial t} & =2 \int_{M} H(x, y, t) f_{i}(y) d y \\
& \leq C(n) \sup _{r \geq \sqrt{t}} \frac{1}{V\left(B_{x}(r)\right)} \int_{B_{x}(r)} f_{i}(y) d y \\
& \leq C(n) \sup _{r \geq \sqrt{t}} k(x, t) .
\end{aligned}
$$

Note that we also have $\left(v_{i}\right)_{t} \geq 0$. Hence

$$
0 \leq \frac{\partial v}{\partial t}(x, t) \leq C(n) \sup _{r \geq \sqrt{t}} k(x, t) \leq \frac{C(n)}{\sqrt{t}} \int_{\sqrt{t}}^{\infty} k(x, s) d s .
$$

All and (1.5), (4.12) and (4.14), for any $t_{0}>1$, and $r>0$, the function $v(x, t)-v\left(0, t_{0}\right)$ is bounded in $B_{0}(r) \in\left[t_{0}-1, t_{0}+1\right]$ by a constant which is independent of $t_{0}$ and

$$
\lim _{t \rightarrow \infty} \sup _{B_{o}(r)}\left|\nabla_{b} v(., t)\right| \rightarrow 0
$$

Hence we know that (ii) is true.

Now we denote that

$$
\omega=u-v .
$$

Lemma 4.2. Assume that $\rho$ satisfies the equality:

$$
\rho_{\alpha, \bar{\beta}, 0}=0
$$

for $\alpha, \beta=1, \cdots, n$. As $t \rightarrow \infty$, we have $\left\|\rho-\sqrt{-1} \partial_{b} \overline{\partial_{b}} \omega\right\|$ converges to zero uniformly on compact subsets of $M$. 
Proof. We claim that

$$
\left\|\rho-i \partial_{b} \overline{\partial_{b}} \omega\right\|(x, t) \leq \int_{M} H(x, y, t)\|\rho\|(y) d y .
$$

If (4.15) is true, then one can using [CF, (3.13)] again to conclude that, for $x \in B_{o}(\sqrt{t})$,

$$
\begin{aligned}
\left\|\rho-i \partial_{b} \overline{\partial_{b}} \omega\right\|(x, t) & \leq C(n) \sup _{r \geq \sqrt{t}} \frac{1}{V\left(B_{x}(r)\right)} \int_{B_{x}(r)}\|\rho\|(y) d y \\
& \leq C(n) \sup _{r \geq \sqrt{t}} \frac{1}{V\left(B_{x}(2 r)\right)} \int_{B_{x}(2 r)}\|\rho\|(y) d y
\end{aligned}
$$

for some positive constant $C(n)$. From the assumption (1.5), this implies that

$$
\sup _{B_{o}(\sqrt{t})}\left\|\rho-i \partial_{b} \overline{\partial_{b}} \omega\right\|(., t) \rightarrow 0
$$

as $t \rightarrow \infty$ and the lemma follows.

To prove (4.15), we first observe that since $\rho$ is real closed $(1,1)$-form and the torsion is vanishing, locally it can be written as $i \partial_{b} \overline{\partial_{b}} \Psi$. Then we have

$$
\triangle_{b} \Psi+i h \Psi_{0}=2 f
$$

where $h=\sum_{\alpha=1}^{n} h_{\alpha \bar{\alpha}}$. From the equation (1.2), we know that the equation (4.16) turns into the following equation

$$
\triangle_{b} \Psi=2 f
$$

From the definition of $\omega$, we have

$$
\left(\triangle_{b}-\frac{\partial}{\partial t}\right)(\Psi-\omega)(x, t)=0 .
$$

Hence $\eta=\rho-i \partial_{b} \bar{\partial}_{b} \omega=i \partial_{b} \bar{\partial}_{b}(\Psi-\omega)$ satisfies the following Lichnerowicz-Laplacian equation

$$
\frac{\partial}{\partial t} \eta_{\alpha \bar{\beta}}=\triangle_{b} \eta_{\alpha \bar{\beta}}+2 R_{\alpha \bar{\gamma} \mu \bar{\beta}} \eta_{\gamma \bar{\mu}}-\left(R_{\gamma \bar{\beta}} \eta_{\alpha \bar{\gamma}}+R_{\alpha \bar{\gamma}} \eta_{\gamma \bar{\beta}}\right) .
$$

Here we use the CR Bochner-Weitzenböck formula as in [CCF. Now by (1.5), since $\omega=0$ at $t=0$, we can have the following inequality

$$
\int_{M}\|\eta\|(x, 0) \exp \left(-a r^{2}(x)\right) d x<\infty
$$

for any $a>0$. Next we estimate $\left|\left(\nabla^{H}\right)^{2} v\right|$. By CR Bochner formula ([CC]), we have

$$
\left(\triangle_{b}-\frac{\partial}{\partial t}\right)\left|\nabla_{b} v\right|^{2}=\triangle_{b}\left|\nabla_{b} v\right|^{2}-\frac{\partial}{\partial t}\left|\nabla_{b} v\right|^{2} \geq 2\left|\left(\nabla^{H}\right)^{2} v\right|^{2}+4\left\langle J \nabla_{b} v, \nabla_{b} v_{0}\right\rangle .
$$


From Theorem 3.1 and vanishing of the torsion in assumptions in Theorem 1.2, we have $v_{0}=0$ for all $t \geq 0$. so the inequality becomes the following

$$
\left(\triangle_{b}-\frac{\partial}{\partial t}\right)\left|\nabla_{b} v\right|^{2} \geq 2\left|\left(\nabla^{H}\right)^{2} v\right|^{2}
$$

For any $T>1$ and $r^{2} \geq T$, multiplying the inequality (4.18) by a suitable cut-off function and integrating by parts, we have

$$
\begin{aligned}
& \int_{0}^{T}\left[\frac{1}{V\left(B_{o}(r)\right)} \int_{B_{o}(r)}\left|\left(\nabla^{H}\right)^{2} v\right|^{2} d x\right] d t \\
\leq & C_{1}\left[\frac{1}{r^{2}} \int_{0}^{T} \frac{1}{V\left(B_{o}(2 r)\right)} \int_{B_{o}(2 r)}\left|\nabla_{b} v\right|^{2} d x d t+\frac{1}{V\left(B_{o}(2 r)\right)} \int_{B_{o}(2 r)}\left|\nabla_{b} u\right|^{2} d x\right] .
\end{aligned}
$$

Then we have

$$
\begin{aligned}
& \int_{0}^{T} \frac{1}{V\left(B_{o}(r)\right)} \int_{B_{o}(r)}\left|\left(\nabla^{H}\right)^{2} v\right|^{2} d x d t \\
\leq & C_{2}\left[(T+1) \frac{1}{V\left(B_{o}(8 r)\right)} \int_{B_{o}(8 r)}\left|\nabla_{b} u\right|^{2} d x\right. \\
& \left.+\int_{0}^{T} t^{-2}\left(\int_{8 r}^{\infty} \exp \left(-\frac{C\left(\rho_{2}\right) s^{2}}{8 t}\right) s \frac{1}{V\left(B_{o}(s)\right)} \int_{B_{o}(s)}\left|\nabla_{b} u\right|^{2} d y d s\right)^{2} d t\right] .
\end{aligned}
$$

From Theorem 2.3. (1.5) and (4.20),

$$
\begin{aligned}
& \int_{0}^{T} \frac{1}{V\left(B_{o}(r)\right)} \int_{B_{o}(r)}\left|\left(\nabla^{H}\right)^{2} v\right|^{2} d t \\
\leq & C_{3}\left[(T+1) \frac{1}{V\left(B_{o}(8 r)\right)} \int_{B_{o}(8 r)}\left|\nabla_{b} u\right|^{2} d x\right. \\
& \left.+\int_{0}^{T}\left(\int_{4 r}^{\infty} \exp \left(-\frac{C\left(\rho_{2} s^{2}\right)}{8 t}\right) d\left(\frac{s^{2}}{t}\right)\right)^{2} d t\right] \\
\leq & C_{4}(T+1)\left[\frac{1}{V\left(B_{o}(4 r)\right)} \int_{B_{o}(4 r)}\left|\nabla_{b} u\right|^{2} d x+1\right] \\
\leq & C_{5}(T+1)\left[\left(\int_{16 r}^{\infty} k(s) d s\right)^{2}+r^{2} \frac{1}{V\left(B_{o}(16 r)\right)} \int_{B_{o}(16 r)}\|\rho\|^{2} d x+1\right] \\
\leq & C_{6}(T+1)\left[r^{2} \frac{1}{V\left(B_{o}(8 r)\right)} \int_{B_{o}(8 r)}\|\rho\|^{2} d x+1\right],
\end{aligned}
$$

where we use Theorem 2.3. We can also obtain from Theorem 2.3 that

$$
\frac{1}{V\left(B_{o}(r)\right)} \int_{B_{o}(r)}\left|\left(\nabla^{H}\right)^{2} u\right|^{2} d x \leq C_{7}\left[r^{2} \frac{1}{V\left(B_{o}(2 r)\right)} \int_{B_{o}(2 r)}\|\rho\|^{2} d x+1\right]
$$


for some constant $C_{7}$ depending only on $n$. From (4.21) and (4.22), we have

$$
\begin{aligned}
& \int_{0}^{T} \frac{1}{V\left(B_{o}(r)\right)} \int_{B_{o}(r)}\|\rho-\sqrt{-1} \partial \bar{\partial} \omega\|^{2} d x d t \\
\leq & C(T+1)\left[r^{2} \frac{1}{V\left(B_{o}(2 r)\right)} \int_{B_{o}(2 r)}\|\rho\|^{2} d x+1\right]
\end{aligned}
$$

for some constant $C$ independent of $T$ and $r$. From (1.6) and (4.23),

$$
\lim _{r \rightarrow \infty} \inf \int_{0}^{T} \int_{B_{o}(r)}\|\eta\|^{2}(x, t) \exp \left(-a r^{2}\right) d x d t<\infty
$$

From (4.17), we have

$$
\left(\triangle_{b}-\frac{\partial}{\partial t}\right)\|\eta\| \geq 0
$$

Therefore we know that $F=\eta-\int_{M} H(x, y, t)\|\eta\|(y, 0) d y$ is also a subsolution of the CR heat equation. Finally, it follow from (4.24) and the proof of [NT2, Theorem 1.2.] and [CCF, Lemma 4.5.] that

$$
\|\eta\| \leq \int_{M} H(x, y, t)\|\eta\|(y, 0) d y
$$

and then (4.15) holds.

\section{Structures of Complete Noncompact Sasakian Manifolds}

In this section, as a consequence of Corollary 1.2 we are able to investigate the structure of complete noncompact CR $(2 n+1)$-manifolds of nonnegative pseudohermitian bisectional curvature and vanishing torsion (Sasakain) and complete steady CR Yamabe solitons as well.

Let $\left\{U_{\alpha}\right\}$ be an open covering of $M$ and $\pi_{\alpha}: U_{\alpha} \rightarrow V_{\alpha} \subset \mathbf{C}^{n}$ submersion. Since $M$ is a Sasakian manifold ([FOW] $)$, we have a transverse Kahler structure $\left(V_{\alpha}, g_{\alpha}^{T}\right)$ as following : There is a canonical isomorphism

$$
d \pi_{\alpha}: D_{p} \rightarrow T_{\pi_{\alpha}(p)} V_{\alpha}
$$

for $D=\operatorname{Ker} \theta \subset T M$ and $p \in U_{\alpha}$. Let $\left\{z^{1,} z^{2}, \ldots, z^{n}\right\}$ be the local holomorphic coordinates on $V_{\alpha}$ and $T_{1,0}(M)=(D \otimes \mathbf{C})^{1,0}$ span by the vectors of the form

$$
Z_{i}=\left(\frac{\partial}{\partial z^{i}}-\theta\left(\frac{\partial}{\partial z^{i}}\right) T\right)
$$

for $i=1,2, \ldots, n$. It is clear that

$$
d \theta\left(Z_{i}, Z_{\bar{j}}\right)=d \theta\left(\frac{\partial}{\partial z^{i}}, \frac{\partial}{\partial z^{\bar{j}}}\right) .
$$

Then the restriction of $d \theta$ to the slice in $U_{\alpha}$ is the Kaehler form which is the same as the Kaehler metric $g_{\alpha}^{T}$

on $V_{\alpha}$. By this expression, we know that $z_{i} f=\frac{\partial f}{\partial z^{i}}$ locally; in other words, we could really view a Sasakian manifold as the disjoint union of the slices on which there are transverse Kahler structures. As an example, 
$\left\{Z_{j}=\frac{\partial}{\partial z^{j}}+i \bar{z}^{j} \frac{\partial}{\partial t} ; T=\frac{\partial}{\partial t}\right\}$ is exactly a local frame and $\theta=d t+i \sum_{j}\left(z^{j} d \bar{z}^{j}-\bar{z}^{j} d z^{j}\right)$ is a pseudohermitian structure on a $(2 n+1)$-dimensional Heisenberg group $H^{n}$.

Note that in our situation as the Corollary 1.2 and Lemma 4.1, the CR plurisuharmonic function

$$
\partial_{b} \bar{\partial}_{b} u \geq 0
$$

is the same of the usual plurisuharmonic function with respect to the transverse Kahler structures on $V_{\alpha}$

$$
\partial \bar{\partial} u \geq 0
$$

if $u_{0}=0$ in a Sasakian manifold.

In this section, we first obtain the following Proposition which served as the CR analogue of results as in [NT1, Theorem 3.1.].

Proposition 5.1. Let $(M, J, \theta)$ be a complete $C R(2 n+1)$-manifold with nonnegative pseudohermitian bisectional curvature and vanishing torsion. Let $u(x)$ be a continous $C R$ plurisubharmonic function on $M$ with $u_{0}=0$. Assume that

$$
|u|(x) \leq C \exp \left(a r^{2}(x)\right)
$$

for some positive constants $a$ and $C$. Let $v(x, t)$ be the solution to the heat equation on $M \times\left[0, \frac{C_{0}}{16 a}\right]$ with initial value $u$, obtained by Theorem 3.1. Then there exists $T_{0}>0$ depending only on a and there exists $T_{0}>T_{1}>0$ such that the following is true:

(i) For $0<t \leq T_{0}, v(., t)$ is a smooth plurisubharmonic function.

(ii) Let

$$
\left.K(x, t)=\left\{\omega \in T_{x}^{1,0} M\right) \mid v_{\alpha \bar{\beta}}(x, t) \omega^{\alpha}=0, \text { for all } \beta\right\}
$$

be the null space of $v_{\alpha \bar{\beta}}$. Then for $0<t<T_{1}, K(x, t)$ is a distribution on $M$.

In order to give an application of Theorem 1.2, we also need the following CR Liouvelle property.

Lemma 5.1. Let $(M, J, \theta)$ be a complete $C R(2 n+1)$-manifold with nonnegative pseudohermitian bisectional curvature and vanishing torsion. Let $u(x)$ be a continuous CR Plurisubharmonic function with $u_{0}=0$ on M. Assume that

$$
u(x)=o(\log r) .
$$

Then $u(x)$ must be a constant. 
Proof. We may assume that $M$ is simply connected. First we let $u_{c}=\max \{u, c\}$ with $v_{c} \geq 0$. By the assumption (5.2), we can conclude that $u_{c}$ satisfies (5.1) and $u_{c}$ is CR plurisubharmonic. Therefore, we can solve the $\mathrm{CR}$ heat equation with $u_{c}(x)$ as the initial data. Denote the solution by $v_{c}$ on $M \times\left[0, T_{0}\right]$. Applying Theorem 5.1(i) we obtain that $v_{c}$ is CR plurisubharmonic. By Proposition 5.1 (ii), for any $t_{0}>0$ small enough, $D=D_{1} \times D_{2}$ isometrically and $\left(v_{c}\right)_{\alpha \bar{\beta}}$ is zero when restricted on $D_{1}$ and $\left(v_{c}\right)_{\alpha \bar{\beta}}$ is positive everywhere when restricted on $D_{2}$. By Corollary 3.1 we have

$$
v_{c}(x, t)=o(\log r(x))
$$

Hence when restricted on $D_{2},(\underline{5.3})$ is still true. This contradicts the fact $\left(\partial_{b} \bar{\partial}_{b} v_{c}\right)^{n}=0([\mathbf{N T 1}$, Lemma 3.3.]) and $\left(v_{c}\right)_{\alpha \bar{\beta}}$ is positive when restricted on $D_{2}$ unless $D=D_{1}$. Hence

$$
\left(v_{c}\right)_{\alpha \bar{\beta}}=0
$$

on $M$ for all $t_{0}>0$ small enough. By the gradient estimate in [BBG], CKT] and (5.3), we can conclude that $\left(v_{c}\right)\left(x, t_{0}\right)$ is a constant, provided $t_{0}>0$ is small enough. Hence $u_{c}$ is constant. Since $c$ is arbitrary, it shows that $u(x)$ is constant.

\section{The proof of Corollary 1.2 :}

Proof. By Theorem 1.2, we can solve $\sqrt{-1} \partial_{b} \bar{\partial}_{b} u=\rho$ where $u$ satisfies (1.7). From (1.8), we have $u(x)=$ $o(\log r)$. We obtain that $u(x)$ is constant by the Lemma 5.1 Then the proof is completed.

Remark 5.1. Let $\left(L, h^{L}\right)$ be a holomorphic line bundle over a non-compact complete kähler manifold $\Omega$, where $h^{L}$ denotes the Hermitian fiber metric of $L$. Assume that the curvature $R^{L}$ induced by $h^{L}$ is positive. Then $M=\left\{v \in L^{*}:|v|_{h^{L^{*}}}^{2}=1\right\}$ is a noncompact Sasakian manifold. This is an interesting example to understand the relation between CR Poincaré-Lelong equation on $M$ and Poincaré-Lelong equation on $\Omega$. We will explore this example in a forthcoming paper.

\section{Acknowledgement}

The authors would like to thank Professor Xiangyu Zhou and the Editorial Board for the invitation to contribute this paper to a special issue of Acta Mathematica Sinica in memory of Professor Qikeng Lu. 


\section{Appendix A.}

We introduce some basic materials in a pseudohermitian $(2 n+1)$-manifold ( see [L1, L2] for more details ). Let $(M, \xi)$ be a $(2 n+1)$-dimensional, orientable, contact manifold with contact structure $\xi$. A CR structure compatible with $\xi$ is an endomorphism $J: \xi \rightarrow \xi$ such that $J^{2}=-1$. We also assume that $J$ satisfies the following integrability condition: If $X$ and $Y$ are in $\xi$, then so are $[J X, Y]+[X, J Y]$ and $J([J X, Y]+[X, J Y])=[J X, J Y]-[X, Y]$.

Let $\left\{T, Z_{\alpha}, Z_{\bar{\alpha}}\right\}$ be a frame of $T M \otimes \mathbb{C}$, where $Z_{\alpha}$ is any local frame of $T_{1,0}, Z_{\bar{\alpha}}=\overline{Z_{\alpha}} \in T_{0,1}$ and $T$ is the characteristic vector field. Then $\left\{\theta, \theta^{\alpha}, \theta^{\bar{\alpha}}\right\}$, which is the coframe dual to $\left\{T, Z_{\alpha}, Z_{\bar{\alpha}}\right\}$, satisfies

$$
d \theta=i h_{\alpha \bar{\beta}} \theta^{\alpha} \wedge \theta^{\bar{\beta}}
$$

for some positive definite hermitian matrix of functions $\left(h_{\alpha \bar{\beta}}\right)$, if we have this contact structure, we call such $M$ a strictly pseudoconvex CR $(2 n+1)$-manifold.

The Levi form $\langle,\rangle_{L_{\theta}}$ is the Hermitian form on $T_{1,0}$ defined by

$$
\langle Z, W\rangle_{L_{\theta}}=-i\langle d \theta, Z \wedge \bar{W}\rangle
$$

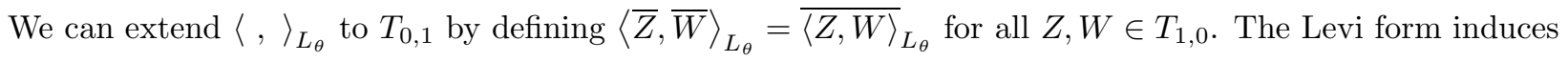
naturally a Hermitian form on the dual bundle of $T_{1,0}$, denoted by $\langle,\rangle_{L_{\theta}^{*}}$, and hence on all the induced tensor bundles. Integrating the Hermitian form (when acting on sections) over $M$ with respect to the volume form $d \mu=\theta \wedge(d \theta)^{n}$, we get an inner product on the space of sections of each tensor bundle.

The pseudohermitian connection of $(J, \theta)$ is the connection $\nabla$ on $T M \otimes \mathbb{C}$ (and extended to tensors) given in terms of a local frame $Z_{\alpha} \in T_{1,0}$ by

$$
\nabla Z_{\alpha}=\theta_{\alpha}^{\beta} \otimes Z_{\beta}, \quad \nabla Z_{\bar{\alpha}}=\theta_{\bar{\alpha}}^{\bar{\beta}} \otimes Z_{\bar{\beta}}, \quad \nabla T=0,
$$

where $\theta_{\alpha}{ }^{\beta}$ are the 1 -forms uniquely determined by the following equations:

$$
\begin{aligned}
d \theta^{\beta} & =\theta^{\alpha} \wedge \theta_{\alpha}{ }^{\beta}+\theta \wedge \tau^{\beta}, \\
0 & =\tau_{\alpha} \wedge \theta^{\alpha}, \\
0 & =\theta_{\alpha}{ }^{\beta}+\theta_{\bar{\beta}}{ }^{\bar{\alpha}} .
\end{aligned}
$$


We can write (by Cartan lemma) $\tau_{\alpha}=A_{\alpha \gamma} \theta^{\gamma}$ with $A_{\alpha \gamma}=A_{\gamma \alpha}$. The curvature of Webster-Stanton connection, expressed in terms of the coframe $\left\{\theta=\theta^{0}, \theta^{\alpha}, \theta^{\bar{\alpha}}\right\}$, is

$$
\begin{aligned}
& \Pi_{\beta}{ }^{\alpha}=\bar{\Pi}_{\bar{\beta}}^{\bar{\alpha}}=d \omega_{\beta}{ }^{\alpha}-\omega_{\beta}{ }^{\gamma} \wedge \omega_{\gamma}{ }^{\alpha}, \\
& \Pi_{0}{ }^{\alpha}=\Pi_{\alpha}{ }^{0}=\Pi_{0}{ }^{\bar{\beta}}=\Pi_{\bar{\beta}}{ }^{0}=\Pi_{0}{ }^{0}=0 .
\end{aligned}
$$

Webster showed that $\Pi_{\beta}{ }^{\alpha}$ can be written

$$
\Pi_{\beta}{ }^{\alpha}=R_{\beta}{ }^{\alpha}{ }_{\rho \bar{\sigma}} \theta^{\rho} \wedge \theta^{\bar{\sigma}}+W_{\beta}{ }^{\alpha}{ }_{\rho} \theta^{\rho} \wedge \theta-W^{\alpha}{ }_{\beta \bar{\rho}} \theta^{\bar{\rho}} \wedge \theta+i \theta_{\beta} \wedge \tau^{\alpha}-i \tau_{\beta} \wedge \theta^{\alpha},
$$

where the coefficients satisfy

$$
R_{\beta \bar{\alpha} \rho \bar{\sigma}}=\overline{R_{\alpha \bar{\beta} \sigma \bar{\rho}}}=R_{\bar{\alpha} \beta \bar{\sigma} \rho}=R_{\rho \bar{\alpha} \beta \bar{\sigma}}, \quad W_{\beta \bar{\alpha} \gamma}=W_{\gamma \bar{\alpha} \beta} .
$$

Here $R_{\gamma}{ }^{\delta}{ }_{\alpha \bar{\beta}}$ is the pseudohermitian curvature tensor, $R_{\alpha \bar{\beta}}=R_{\gamma}{ }^{\gamma}{ }_{\alpha \bar{\beta}}$ is the pseudohermitian Ricci curvature tensor, $S=R_{\alpha \bar{\alpha}}$ is the Tanaka-Webster scalar curvature and $A_{\alpha \beta}$ is the torsion tensor. Furthermore, we define the bisectional curvature

$$
R_{\alpha \bar{\alpha} \beta \bar{\beta}}(X, Y)=R_{\alpha \bar{\alpha} \beta \bar{\beta}} X_{\alpha} X_{\bar{\alpha}} Y_{\beta} Y_{\bar{\beta}}
$$

and the bitorsion tensor

$$
T_{\alpha \bar{\beta}}(X, Y):=i\left(A_{\bar{\beta} \bar{\rho}} X_{\rho} Y_{\alpha}-A_{\alpha \rho} X_{\bar{\rho}} Y_{\bar{\beta}}\right)
$$

and the torsion tensor

$$
\operatorname{Tor}(X, Y):=h^{\alpha \bar{\beta}} T_{\alpha \bar{\beta}}(X, Y)=i\left(A_{\bar{\alpha} \bar{\rho}} X_{\rho} Y_{\alpha}-A_{\alpha \rho} X_{\bar{\rho}} Y_{\bar{\alpha}}\right)
$$

for any $X=X_{\bar{\alpha}} Z_{\alpha}, Y=Y_{\bar{\alpha}} Z_{\alpha}$ in $T_{1,0}$.

We will denote components of covariant derivatives with indices preceded by comma; thus write $A_{\alpha \beta, \gamma}$. The indices $\{0, \alpha, \bar{\alpha}\}$ indicate derivatives with respect to $\left\{T, Z_{\alpha}, Z_{\bar{\alpha}}\right\}$. For derivatives of a scalar function, we will often omit the comma, for instance

$$
u_{\alpha}=Z_{\alpha} u ; u_{\alpha \bar{\beta}}=Z_{\bar{\beta}} Z_{\alpha} u-\omega_{\alpha}^{\gamma}\left(Z_{\bar{\beta}}\right) Z_{\gamma} u
$$

For a smooth real-valued function $u$, the subgradient $\nabla_{b}$ is defined by $\nabla_{b} u \in \xi$ and $\left\langle Z, \nabla_{b} u\right\rangle_{L_{\theta}}=d u(Z)$ for all vector fields $Z$ tangent to contact plane. Locally

$$
\nabla_{b} u=\sum_{\alpha} u_{\bar{\alpha}} Z_{\alpha}+u_{\alpha} Z_{\bar{\alpha}}
$$

and

$$
u_{0}=T u
$$


We can use the connection to define the subhessian as the complex linear map

$$
\left(\nabla^{H}\right)^{2} u: T_{1,0} \oplus T_{0,1} \rightarrow T_{1,0} \oplus T_{0,1}
$$

by

$$
\left(\nabla^{H}\right)^{2} u(Z)=\nabla_{Z} \nabla_{b} u
$$

In particular,

$$
\left|\nabla_{b} u\right|^{2}=2 u_{\alpha} u_{\bar{\alpha}}, \quad\left|\nabla_{b}^{2} u\right|^{2}=2\left(u_{\alpha \beta} u_{\bar{\alpha} \bar{\beta}}+u_{\alpha \bar{\beta}} u_{\bar{\alpha} \beta}\right) .
$$

Also

$$
\Delta_{b} u=\operatorname{Tr}\left(\left(\nabla^{H}\right)^{2} u\right)=\sum_{\alpha}\left(u_{\alpha \bar{\alpha}}+u_{\bar{\alpha} \alpha}\right) .
$$

The Kohn-Rossi Laplacian $\square_{b}$ on functions is defined by

$$
\square_{b} \varphi=2 \bar{\partial}_{b}^{*} \bar{\partial}_{b} \varphi=\left(\Delta_{b}+i n T\right) \varphi=-2 \varphi_{\bar{\alpha}}^{\bar{\alpha}}
$$

and on $(p, q)$-forms is defined by

$$
\square_{b}=2\left(\bar{\partial}_{b}^{*} \bar{\partial}_{b}+\bar{\partial}_{b} \bar{\partial}_{b}^{*}\right)
$$

Next we recall the following commutation relations ([L1]). Let $\varphi$ be a scalar function and $\sigma=\sigma_{\alpha} \theta^{\alpha}$ be a $(1,0)$ form, then we have

$$
\begin{aligned}
\varphi_{\alpha \beta} & =\varphi_{\beta \alpha}, \\
\varphi_{\alpha \bar{\beta}}-\varphi_{\bar{\beta} \alpha} & =i h_{\alpha \bar{\beta}} \varphi_{0}, \\
\varphi_{0 \alpha}-\varphi_{\alpha 0} & =A_{\alpha \beta} \varphi_{\bar{\beta}}, \\
\sigma_{\alpha, 0 \beta}-\sigma_{\alpha, \beta 0} & =\sigma_{\alpha, \bar{\gamma}} A_{\gamma \beta}-\sigma_{\gamma} A_{\alpha \beta, \bar{\gamma}}, \\
\sigma_{\alpha, 0 \bar{\beta}}-\sigma_{\alpha, \bar{\beta} 0} & =\sigma_{\alpha, \gamma} A_{\bar{\gamma} \bar{\beta}}+\sigma_{\gamma} A_{\bar{\gamma} \bar{\beta}, \alpha},
\end{aligned}
$$

and

$$
\begin{aligned}
\sigma_{\alpha, \beta \gamma}-\sigma_{\alpha, \gamma \beta} & =i A_{\alpha \gamma} \sigma_{\beta}-i A_{\alpha \beta} \sigma_{\gamma} \\
\sigma_{\alpha, \bar{\beta} \bar{\gamma}}-\sigma_{\alpha, \bar{\gamma} \bar{\beta}} & =i h_{\alpha \bar{\beta}} A_{\bar{\gamma} \bar{\rho}} \sigma_{\rho}-i h_{\alpha \bar{\gamma}} A_{\bar{\beta} \bar{\rho}} \sigma_{\rho}, \\
\sigma_{\alpha, \beta \bar{\gamma}}-\sigma_{\alpha, \bar{\gamma} \beta} & =i h_{\beta \bar{\gamma}} \sigma_{\alpha, 0}+R_{\alpha \bar{\rho} \beta \bar{\gamma}} \sigma_{\rho} .
\end{aligned}
$$

Moreover for multi-index $I=\left(\alpha_{1}, \ldots, \alpha_{p}\right), \bar{J}=\left(\bar{\beta}_{1}, \ldots, \bar{\beta}_{q}\right)$, we denote $I\left(\alpha_{k}=\mu\right)=\left(\alpha_{1}, \ldots, \alpha_{k-1}, \mu, \alpha_{k+1}, \ldots, \alpha_{p}\right)$. Then

$$
\begin{aligned}
\eta_{I \bar{J}, \mu \lambda}-\eta_{I \bar{J}, \lambda \mu}= & i \sum_{k=1}^{p}\left(\eta_{I\left(\alpha_{k}=\mu\right) \bar{J}} A_{\alpha_{k} \lambda}-\eta_{I\left(\alpha_{k}=\lambda\right) \bar{J}} A_{\alpha_{k} \mu}\right) \\
& -i \sum_{k=1}^{q}\left(\eta_{I \bar{J}\left(\bar{\beta}_{k}=\bar{\gamma}\right)} h_{\bar{\beta}_{k} \mu} A_{\lambda}^{\bar{\gamma}}-\eta_{I \bar{J}\left(\bar{\beta}_{k}=\bar{\gamma}\right)} h_{\bar{\beta}_{k} \lambda} A_{\mu}^{\bar{\gamma}}\right),
\end{aligned}
$$


and

$$
\begin{aligned}
\eta_{I \bar{J}, \lambda \bar{\mu}}-\eta_{I \bar{J}, \bar{\mu} \lambda} & =i h_{\lambda \bar{\mu}} \eta_{I \bar{J}, 0}+\sum_{k=1}^{p} \eta_{I\left(\alpha_{k}=\gamma\right) \bar{J}} R_{\alpha_{k}}^{\gamma}{ }_{\lambda \bar{\mu}}+\sum_{k=1}^{q} \eta_{I \bar{J}\left(\bar{\beta}_{k}=\bar{\gamma}\right)} R_{\bar{\beta}_{k}}{ }^{\bar{\gamma}}{ }_{\lambda \bar{\mu}} \\
\eta_{I \bar{J}, 0 \mu}-\eta_{I \bar{J}, \mu 0} & =A_{\mu}^{\bar{\rho}} \eta_{I \bar{J}, \bar{\rho}}-\sum_{k=1}^{p} A_{\alpha_{k} \mu, \bar{\rho}} \eta_{I\left(\alpha_{k}=\rho\right) \bar{J}}+\sum_{k=1}^{q} A_{\mu \rho, \bar{\beta}_{k}} \eta_{I \bar{J}\left(\bar{\beta}_{k}=\bar{\rho}\right)} .
\end{aligned}
$$

\section{REFERENCES}

[BBG] F. Baudoin, M. Bonnefont, N. Garofalo, A sub-Riemannian curvature-dimension inequality, volume doubling property and the Poincare inequality, Math. Ann. 358 (2014), no. 3-4, 833-860.

[CaC] O. Calin and D.-C. Chang, Sub-Riemannian Geometry: General Theory and Examples, Encyclopedia of mathematics and its applications, 126, Cambridge University Press, Cambridge-New York-Melbourne, 2009.

[CCFI] O. Calin, D.-C. Chang, K. Furutani and C. Iwasaki, Heat Kernels for Elliptic and Sub-elliptic Operators: Methods and Techniques, Birkhäuser-Verlag, Boston, Massachusetts, 2010.

[CCC] Huai-Dong Cao, S.-C. Chang and Chi-Wei Chen, On three-dimensional CR Yamabe solitons, to appear in JGEA.

[CCF] S.-C. Chang, T.-H. Chang and Y.-W. Fan, Linear Trace Li-Yau-Hamilton inequality for the CR Lichnerowicz-Laplacian heat equation, J. Geom. Anal. 25 (2015): 783-819.

[CCHT] D.-C. Chang, S.-C. Chang, Y.-B. Han and J.-Z. Tie, A CR Analogue of Yau's Conjecture On pseudoharmonic functions of polynomial growth, submitted, 2017.

[CC] S.-C Chang, H.-L. Chiu, Nonnegativity of CR Paneitz operator and its application to CR Obata's theorem in a pseudohermitian $(2 n+1)$-manifold, J. Geom. Anal. 19 (2009), 261-287.

[CF] S.-C Chang, Y.-W. Fan, An optimal gap theorem in a complete strictly pseudoconvex CR $(2 n+1)$-Manifold, J. Geom. Anal. 26 (2016): 2425-2449.

[CHL] S.-C. Chang, Y.-B. Han and C. Lin, On sharp dimension estimates of CR holomorphic functions, preprint

[CKL] S.-C. Chang, T.-J. Kuo and S.-H. Lai, Li-Yau Gradient Estimate and Entropy Formulae for the CR heat equation in a Closed Pseudohermitian 3-manifold, J. Geom. Anal. 89 (2011), 185-216.

[CKT] S.-C. Chang, T.-J. Kuo and J.-Z. Tie, Yau's gradient estimate and Liouville Theorem for positive pseudo-harmonic functions in a complete pseudo-Hermitian manforld, arXiv:1506.03270.

[CTZ] B.-L. Chen, S.-H. Tang and X.-P. Zhu, A uniformization theorem for complete non-compact Kaehler surfaces with positive bisectional curvature. J. Diff. Geom. 67 (3) (2004), 519-570.

[CZ] Bing-Long Chen and Xi-Ping Zhu, On complete noncompact Kaehler manifolds with positive bisectional curvature, Math. Ann. 327 (2003), 1-23.

[CH] W. L. Chow, Uber Systeme van Linearen partiellen Differentialgleichungen erster Ordnung, Math. Ann., 117 (1939), 98-105.

[D] Harold Donnelly, Uniqueness of positive solutions of the heat equation, AMS, 99, No. 2, 1987.

[E] Lawrence C. Evans, Partial differential equations. Graduate studies in math. 19, Amer. Math. Soc., 2010.

[FOW] A. Futaki, H. Ono and G.-F. Wang, Transverse Kahler geometry of Sasaki manifolds and toric Sasaki-Einstein manifolds, J. Diff. Geom., 83 (2009) 585-635 
[FS] G.B. Folland and E.M. Stein, Estimates for the $\bar{\partial}_{b}$ complex and analysis on the Heisenberg group, Comm. Pure Appl. Math. 27 (1974), 429-522.

[GV] Ugo Gianazza and Vincenzo Vespri, Analytic semigroups generated by square Hörmander operators, Rend. Istit. Mat. Univ. Trieste Suppl. 28 (1997), 201-220.

[GV2] Ugo Gianazza and Vincenzo Vespri, Generation of analytic semigroups by degenerate elliptic operators, NoDEA, 4 (1997), 305-324.

[GW1] R.-E. Greene and H. Wu, H., Analysis on non-compact Kaehler manifolds, Proc. Symp. Pure. Math. 30, Part II, Amer. Math. Soc. (1977).

[GW2] R.-E. Greene and H. Wu, Gap theorems for noncompact Riemannian manifolds. Duke Math. J. 49 (3) (1982), $731-756$.

[GW3] R.E. Green, H. Wu, Integrals of subharmonic functions on manifolds on negative curvature, Invent. Math. 27 (1974), 265-298

[Gr] A. Greenleaf, The first eigenvalue of a sublaplacian on a pseudohermitian manifold, Comm. Part. Diff. Equ. 10 (2) (1985), 191-217.

[J1] D.S. Jerison, The Dirichlet problem for the Kohn Laplacian on the Heisenberg group, I and II, J. Functional Anal. 43 (1981), 97-141 and 429-522.

[J2] D.S. Jerison, Boundary regularity in the Dirichlet problem for $\square_{b}$ on CR manifolds, C.P.A.M. XXXVI (1983), $143-181$.

[LA] Alessandra Lunardi, Analytic semigroups and optimal regularity in parabolic problems, PNLDE 16, Birkhäuser, Berlin, (1995).

[Li] P.Li, Geometry analysis, Cambridge Studies in Advanced Mathematics, 134, Cambridge University Press, Cambridge, (2012).

[L1] J. M. Lee, Pseudo-Einstein Structure on CR Manifolds, Amer. J. Math. 110 (1988), 157-178.

[L2] J. M. Lee, The Fefferman Metric and Pseudohermitian Invariants, Trans. Amer. Math. Soc. 296 (1986), 411-429.

[LT] P. Li. and L.-F. Tam, The heat equation and harmonic maps of complete manifolds, Invent. math. 105 (1991) 1-46.

[LY] P. Li and S.-T. Yau, On the parabolic kernel of the Schrödinger operator, Acta Math. 156 (1986) 153-201.

[M] Isidro H. Munive, Stochastic completeness and volume growth in sub-Riemannian manifolds, Manuscripta Math. 138 (2012), 299-313.

[Mo] C. Morrey, Multiple Integrals in Calculus of Variations. Springer-Verlag, New York, (1966).

[Mok1] N. Mok, The uniformization theorem for compact Kähler manifolds of nonnegative holomorphic bisectional curvature, J. Diff. Geom. 27 (1988), 179-214.

[Mok2] N. Mok, An embedding theorem of complete Kähler manifolds of positive bisectional curvature onto affine algebraic varieties, Bull. Soc. Math. France. 112 (1984),197-258.

[MR] R.R. Miner, Spherical CR manifolds with amenable holonomy, Int. J. Math. 1 (1990), 479-510.

[MSY] N. Mok, Y.-T. Siu and S.-T. Yau, The Poincaré-Lelong equation on complete Kähler manifolds. Compos. Math. 44 (1981), 183-218.

[N1] L. Ni, An Optimal Gap Theorem, Invent Math. 189 (2012), 737-761 and Erratum.

[N2] L. Ni, Vanishing theorems on complete Kahler manioflds and their applications. J. Diff. Geom. 50 (1998), 89-122. 
[NST] L. Ni, Y. Shi and L.-F. Tam, Poisson equation, Poincare-Lelong equation and curvature decay on complete Kähler manifolds, J. Diff. Geom. 57 (2001) 339-388.

[NT1] L. Ni and L.-F. Tam, Plurisubharmonic functions and the structure of complete Kähler manifolds with nonnegative curvature. J. Diff. Geom. 64 (3) (2003), 457-524.

[NT2] L. Ni and L.-F. Tam, Kähler-Ricci flow and Poincare-Lelong equation, Comm. Anal. Geom. 12 (1) (2004), 111-114.

[S] Y.-T. Siu, Pseudoconvexity and the problem of Levi, Bull. Amer Math. Soc. 84 (1978), 481-512.

[Y] S.-T. Yau, Problem Section, Seminar on Diff. Geom. edit by S. T. Yau, Princeton Univ. Press, 1982.

Department of Mathematics and Statistics, Georgetown University, Washington D. C. 20057, USA, Department of Mathematics, Fu Jen Catholic University, Taipei 242, Taiwan, R.O.C.

E-mail address: chang@georgetown.edu

Department of Mathematics and Taida Institute for Mathematical Sciences (Tims), National Taiwan UniverSity, Taipei 10617, Taiwan, R.O.C., Current address : Yau Mathematical Sciences Center, Tsinghua University, Beijing, China

E-mail address: scchang@math.ntu.edu.tw

School of Mathematics and Statistics, Xinyang Normal University, Xinyang,464000, Henan, P.R. China

E-mail address: yingbohan@163.com

Department of Mathematics, National Tsing Hua University, Hsinchu 30013, Tainan, R.O.C

E-mail address: r97221009@ntu.edu.tw 